\title{
Automated data analysis for static structural health monitoring of masonry heritage structures
}

\author{
Nirvan Makoond ${ }^{1}$ | Luca Pelà ${ }^{1}$ | Climent Molins ${ }^{1}$ | Pere Roca ${ }^{1}$ | Daniel Alarcón ${ }^{1}$
}

${ }^{1}$ Universitat Politècnica de Catalunya (UPC-BarcelonaTech)

\section{Correspondence}

Nirvan Makoond, Department of Civil and Environmental Engineering, Universitat Politècnica de Catalunya (UPC-BarcelonaTech), Jordi Girona 1-3, 08034, Barcelona, Spain

Email: nirvan.makoond@upc.edu

\section{Funding information}

Ajuntament de Sant Cugat through a project aimed at monitoring the Monastery of Sant Cugat, ref. num. C-10764.

Ministry of Education, Culture and Sports of the Spanish Government through a project aimed at studying the structural condition of Mallorca Cathedral, ref. num. 2/131400106ca 5/030300592 EF.

AGAUR agency of the Generalitat de Catalunya and European Social Fund through a predoctoral grant awarded to the corresponding author.

Ministry of Science, Innovation and Universities of the Spanish

Government and European Regional Development Fund through the SEVERUS project, ref. num. RTI2018-099589-B-100.

\begin{abstract}
Summary
Masonry heritage structures are often affected by slow irreversible deterioration mechanisms that can jeopardise structural stability in the foreseeable future. Static structural health monitoring (SHM), aimed at the continuous measurement of key slow-varying parameters, has the potential to identify such mechanisms at a very early stage. This can greatly facilitate the implementation of adequate preventive and remedial measures which can be critical to ensure that such structures are preserved for generations to come. However, since monitored parameters usually experience reversible seasonal variations of the same order of magnitude as changes caused by active mechanisms, identification of the latter is often a difficult task. This paper presents a fully integrated automated data analysis procedure for complete static SHM systems utilising dynamic linear regression models to filter out the effects caused by environmental variations. The method does not only produce estimated evolution rates but also classifies monitored responses in pre-defined evolution states. The procedure has successfully been used to identify vulnerable areas in two important medieval heritage structures in Spain, namely the cathedral of Mallorca and the church of the monastery of Sant Cugat.
\end{abstract}

\section{Keywords}

masonry, heritage, medieval structures, environmental effects, dynamic linear models, ARX

Link to formal publication: https ://doi.org/10.1002/stc.2581

\section{1 | INTRODUCTION}

A large number of the established cultural heritage sites worldwide are made of masonry and the common responsibility to safeguard them for future generations is now widely recognized. Although many of these old buildings prove their structural soundness by surviving to the present time in relatively good condition, many have suffered from considerable damages caused either by natural or man induced events throughout their history. In order to ensure their survival, an accurate evaluation of their current structural condition is often of paramount importance. However, recurrent uncertainties regarding material properties and the complex interaction among structural elements often makes the evaluation of their structural safety challenging, despite recent advances in structural analysis, inspection, testing and monitoring techniques.

Structural health monitoring (SHM), which aims to gain knowledge of the integrity of in-service structures by monitoring damage sensitive features, can prove to be a very useful tool to better comprehend underlying causes of damage in structures. This is particularly true for heritage structures since the extraction of a comprehensive set of samples for laboratory testing is often not feasible due to the heritage value of the structure. Moreover, excessive extensive interventions are undesirable in such cases due to the need to respect authenticity and "a correct diagnosis is [thus] indispensable for a proper evaluation of 
safety and for rational decisions on any treatment measures to be adopted" ${ }^{1}$. Data from SHM can thus prove to be extremely valuable, particularly when ongoing deterioration mechanisms are still present.

In general, monitoring strategies to be applied can be categorised as static or dynamic. Dynamic monitoring is oriented to the characterisation and control of dynamic properties such as natural frequencies, mode shapes and damping ratios ${ }^{2-7}$. Static monitoring is aimed the continuous measurement of slow-varying parameters over a long period ${ }^{8}$. Due to the slow progression of parameters of interest and because the data is largely influenced by seasonal cycles, monitoring periods of at least 2 years are usually required for meaningful conclusions to be derived from the data when it comes to static monitoring. In fact, due to the inability of commonly used analysis techniques to consider several factors and quantify uncertainties related to the analysis, longer monitoring periods are often required to establish a satisfactory level of confidence on resulting conclusions. Although recent research has focused more on data analysis for dynamic monitoring ${ }^{9-14}$, presumably due to the fact that this monitoring strategy enables the extraction of useful information about the structure as a whole in a short time period, it must be said that masonry heritage structures are most often affected by slow ongoing deterioration mechanisms that are not easily identifiable. As such, static monitoring appears to be particularly appealing.

Since unreinforced masonry is characterised by a very low tensile strength, cracking is probably the most common pathology visible in masonry structures of the built cultural heritage. Because structural integrity can be impaired if cracks remain active, crack monitoring has emerged as one of the most important basic parameters in long-term heritage SHM systems ${ }^{15}$. Since lateral displacements or rigid rotations of key elements can also severely compromise structural performance, the inclination of such elements and distances between them are the other structural parameters that are most often also of interest.

In the case of static SHM, it is essential to remove any anomalies present in the data that are not caused by a physical phenomena related to structural behaviour before any further analysis can be carried out. These often appear as "spikes" in sensor data ${ }^{16}$ and can originate from several sources such as capacitive or inductive noise in the analog signal path, communication errors ${ }^{17}$ or undesired external interactions with the sensor. The initial interpretation task then involves the identification of either a stationary or an evolutionary condition from the recorded data of each monitored response. Although this might appear simple in theory, in actual practice it proves to be difficult ${ }^{18}$, given that features monitored for their sensitivity to damage are also sensitive to changes caused by environmental and operational conditions. In fact, it can even be said that this is a major issue prohibiting the extension of SHM technologies to structures in operation in the real world ${ }^{19}$.

Nevertheless, there exists a number of data normalisation techniques that can be used to separate measurements relevant to structural damage from those associated with the environmental variation of the system ${ }^{20}$. In fact, there are several examples of static monitoring systems applied to masonry cultural heritage structures as shown in Table 1.

TAB LE 1 Some notable examples of static SHM systems installed in masonry cultural heritage structures.

\begin{tabular}{|c|c|c|c|c|}
\hline Structure & $\begin{array}{c}\text { Monitoring } \\
\text { start }\end{array}$ & $\begin{array}{l}\text { No. of } \\
\text { years* }\end{array}$ & $\begin{array}{c}\text { No. of } \\
\text { instruments }\end{array}$ & $\begin{array}{l}\text { Combined with } \\
\text { dynamic tests? }\end{array}$ \\
\hline Santa Maria del Fiore (Opera del Duomo system) 21;22 & 1955 & 54 & 22 & No \\
\hline Santa Maria del Fiore (ISMES system) ${ }^{21 ; 22}$ & 1987 & 20 & 150 & No \\
\hline Basilica of San Marco 23 & 1991 & 3.5 & 23 & No \\
\hline Metropolitan Cathedral in Mexico City ${ }^{24}$ & 1994 & 10 & 38 & Yes \\
\hline Basilica S. Maria Gloriosa dei Frari ${ }^{23}$ & 2003 & 3.6 & 11 & No \\
\hline Cathedral of Modena 25 & 2003 & 9 & 22 & No \\
\hline "Regina Montis Regalis" Basilica of Vicoforte 26 & 2004 & 10 & 133 & No \\
\hline Monastery of Jerónimos 27 & 2005 & 9 & 10 & Yes \\
\hline Roman Arena of Verona ${ }^{28}$ & 2011 & $>4$ & 24 & Yes \\
\hline Church of the Major Seminary of Comillas ${ }^{29}$ & 2012 & 5 & 67 & No \\
\hline
\end{tabular}

* Minimum number of monitoring years (as reported in literature).

As shown, some recent static SHM systems have also been used in conjunction with ambient vibration testing (AVT) and/or dynamic monitoring. Examples of structures where such systems have been used include the monastery of Jéronimos in Lisbon ${ }^{27}$, the Roman Arena ${ }^{28}$ and the stone tomb of Cansignorio 
della Scala ${ }^{30}$ in Verona as well as the Anime Sante church in L'Aquila ${ }^{31}$.

Many of the above-mentioned investigations have relied on two basic procedures for the analysis and interpretation of the data collected from the static monitoring system. The first of which involves carrying out a simple linear regression from the recorded evolution of structural parameters such as crack widths and inclinations (response variables). If an underlying trend is found, the slope of the trend line allows the rate at which the phenomena in question is progressing to be estimated. The second procedure involves examining the correlation between temperature variations and the evolution of each response variable. In certain cases ${ }^{21 ; 23}$, analyses have attempted to remove the underlying periodicity present in crack width evolutions by examining the autocorrelation function of the signal and subtracting a sinusoidal function containing the signal's main period. Although it is true that a periodic sinusoid component fitted to the data is likely to contain the main seasonal behaviour, this theoretical formulation does not explicitly address the effect that measured environmental parameters (predictors) such as temperature or humidity have on the variation of monitored structural parameters. As such, careful implementation is required to avoid components not necessarily caused by seasonal variations from also being removed from the original signal during this processing step. Moreover, in this era of climate change, seasonal variations are becoming less predictable. Therefore it can no longer be assumed that their effects can always be accurately modelled by a regular periodic function.

In order to provide a systematic way of dealing with seasonal changes, a simple method based on the evaluation of reference quantities was proposed ${ }^{18}$. In fact, this method can be considered as one aiming to solve the well-studied problem of identifying directionality from a time series, whereby directionality is defined as asymmetry in time ${ }^{32}$. As such, although it has been successfully applied to analyse data in a few heritage structures ${ }^{18 ; 3}$, like many methods developed to detect directionality in other fields of study ${ }^{34}$, it cannot explicitly consider the effect of measured predictors on the response parameters.

Some authors have intentionally searched for methods that do not require the measurement of environmental variables, arguing that with approaches having this requirement, all factors influencing the parameters of interest must also be monitored and understood in order to have reasonable confidence in any model prediction capability ${ }^{35}$. As a consequence, a method based on finding a stationary linear combination among monitored responses, known as cointegration, has been successfully applied to eliminate the adverse influence of environmental changes from dynamic SHM data whilst maintaining sensitivity to structural damage ${ }^{35}$. However, although such combinations can often be found between two identified natural frequencies, the local nature of response variables in static SHM systems means that finding such combinations can be difficult. Recently, an enhancement of the cointegration approach has been proposed making it more suitable to confronting cases when nonlinear relations between system responses exist ${ }^{9}$. However, since it is based on a Bayesian machine learning approach known as Gaussian process regression, it requires a training period that does not contain any data corresponding to damage ${ }^{9}$. In fact, this is also a requirement of many other sophisticated analysis methods that have been applied to damage detection in the presence of environmental variability from dynamic SHM data. These include negative selection ${ }^{10}$, other machine learning techniques ${ }^{11 ; 19}$ as well as those based on linear and kernel principal component analysis (PCA) ${ }^{12 ; 13 ; 36}$ or on the Mahalanobis squared-distance ${ }^{14}$. This is a difficult requirement when it comes to static SHM systems for masonry heritage structures since the damage phenomena of interest very often relate to very slow and long processes which have begun long before any decision on monitoring could be taken. Moreover, for such cases, previous studies have shown that temperature is clearly one of the most influential environmental factors contributing to the seasonal variations of the local response parameters being monitored. As such, since temperature monitoring can easily be included in any modern monitoring system with very little additional cost, the current research will focus on a method which takes advantage of measured predictors.

One of the simplest approaches which explicitly takes measured environmental variables into consideration was used for filtering out the effect of temperature from crack widths monitored as part of the static monitoring system in the dome of Santa Maria del Fiore ${ }^{22}$. The method attempts to remove the periodicity of the response variable caused by the predictor by simply subtracting the corresponding effect from the identified general linear trend found between the two. A key assumption behind this method is that a linear relationship exists between the selected structural and environmental parameters even if this is not always an adequate representation. Moreover, it can be assumed that due to the thermal inertia of the material, among other factors, the crack width at any point in time will in fact depend not 
only on the current temperature but also on previous ones. Another limitation of this method is that only a single predictor can be used to filter out the environmental variability of the response variable.

Nevertheless, meaningful conclusions on the state of the structure in question could still be derived from the data of all the previously mentioned static monitoring systems. Indeed, these examples show that static SHM can prove to be a powerful tool with respect to the diagnosis of heritage structures. It must be said however, that in every case, expertise and previous experience of the interpreter still play a vital role in the ability to draw conclusions from the processed data. Moreover, most of the procedures that have been applied to the analysis of data from static SHM systems of heritage structures provide no means of adequately quantifying the uncertainties or understanding the confidence levels associated to the estimated trends.

Utilising statistical black box models could prove to be an appealing alternative to remove the effects of environmental factors on measurements since they can exploit a large number of observations to reconstruct dependencies between recorded parameters. In particular, dynamic linear regression models able to represent response variables when they depend linearly on their own rate of change, on the rate of change of predictors as well as on the present value of the predictors appear to be ideal. Although there exists some examples ${ }^{37-39}$ which make use of such models to filter out environmental effects on the evolution of natural frequencies extracted from dynamic monitoring systems, application to static SHM systems has been very limited. In fact, one of the only examples available in literature involves application to the monitoring of a crack on frescos present in Battuti Hall of Conegliano cathedral ${ }^{40}$. In this case, a Single Input-Single Output (SISO) model that comprehends an Auto-Regressive output and an eXogenous input (ARX) was used. Unlike the case of simple linear regression, the dynamic nature of these regression models mean that they are well suited to model complex environmental effects such as those due to thermal inertia.

Although the quality criteria presented in previous research ${ }^{37 ; 40 ; 41}$ can facilitate the choice of adequate ARX model orders, a systematic methodological procedure for the implementation of such models to the analysis of data from complete masonry heritage static SHM systems is still missing from literature. Moreover, to the best of the authors knowledge, Multiple Input-Single Output (MISO) ARX models incorporating both interior and exterior temperatures as predictors have not yet been applied to the case of static SHM systems. Utilising such models could theoretically allow the identified models to better represent the environmental variability imposed on the response variables since they would be able to incorporate effects caused by thermal gradients. In addition, it should also be noted that despite the theoretical advantages of this method over some of the more traditional analysis techniques, application to large data sets from whole networks of sensors in complex heritage structures is still lacking in literature. It is only through such applications that a well-defined systematic procedure can be developed to move from the analysis results to the diagnosis of the structure as a whole.

As such, the current research work aims to develop a holistic automated procedure which includes not only steps on how to implement ARX models to filter out environmental variability for entire static SHM systems installed in masonry heritage structures, but also steps to facilitate the interpretation of results from such analyses towards the overall diagnosis of the structure being monitored.

Before presenting the proposed analysis methodology, a series of simple and intuitive methods that have previously been applied to analyse data from static SHM of masonry heritage structures are presented. Every methodology described is then applied to data from two case studies, namely the Cathedral of Mallorca (monitored from 2003 to 2008) and the church of the monastery of Sant Cugat close to Barcelona (ongoing monitoring since 2017). A comparison of the outcomes that can be derived from the proposed procedure to those obtained from the more intuitive methods presented is also carried out. The results reveal that the proposed automated data analysis procedure can greatly facilitate the prognosis of masonry heritage structures.

\section{2 | PREVIOUSLY APPLIED DATA ANALYSIS METHODOLOGIES}

\section{1 | Linear and nonlinear regression of time-series}

The most intuitive analysis methodology applied to static SHM involves fitting each data set to a linear trend line using ordinary least squares regression, as shown in Figure 1. The slope of the identified trend 
line is then used as an estimate of the evolution rate of the structural parameter in question. Although this method has been used successfully in the past to assess ongoing damage mechanisms in several heritage structures $22 ; 24 ; 31 ; 42$, it can easily be biased by asymmetry caused by the position of the monitoring period in relation to seasonal cycles. Moreover, since it involves fitting a straight-line to an evolution which is clearly not linear, it provides no means of assessing the quality of the fit.

A more appropriate approach to the problem involves evaluating the underlying trend of each response variable after subtracting a sinusoidal function containing the signal's main period. Several methods can be used for identifying such a sinusoidal function. In the context of this research, a method previously employed for analysing data from the static SHM system installed in Mallorca cathedral from 2003 to 2008 was employed. The method makes use of the Levenberg-Marquardt algorithm ${ }^{43}$ to solve the nonlinear least squares problem of fitting the data from each sensor to the following nonlinear function comprising of a periodic component $(A \sin (P t-\phi))$ and a linear one $(B t+C)$.

$$
y=A \sin (P t-\phi)+B t+C
$$

Where $y$ is the monitored structural parameter of interest, $t$ is time while $A, P, \phi, B$ and $C$ are unknowns found using the Levenberg-Marquardt algorithm. Note that $A, P$ and $\frac{\phi}{P}$ correspond respectively to the amplitude, period and phase shift of the periodic component of the function while $B$ and $C$ correspond respectively to the slope and $y$-intercept of the straight line component.

Hence, after the fitting procedure, the evolution of each response variable is modelled by a nonlinear periodic function, as shown in Figure 1. The resulting value of $B$ from Equation (1) is then an estimate of the evolution rate of the monitored structural parameter after removing the identified sinusoidal component containing the signal's main period.

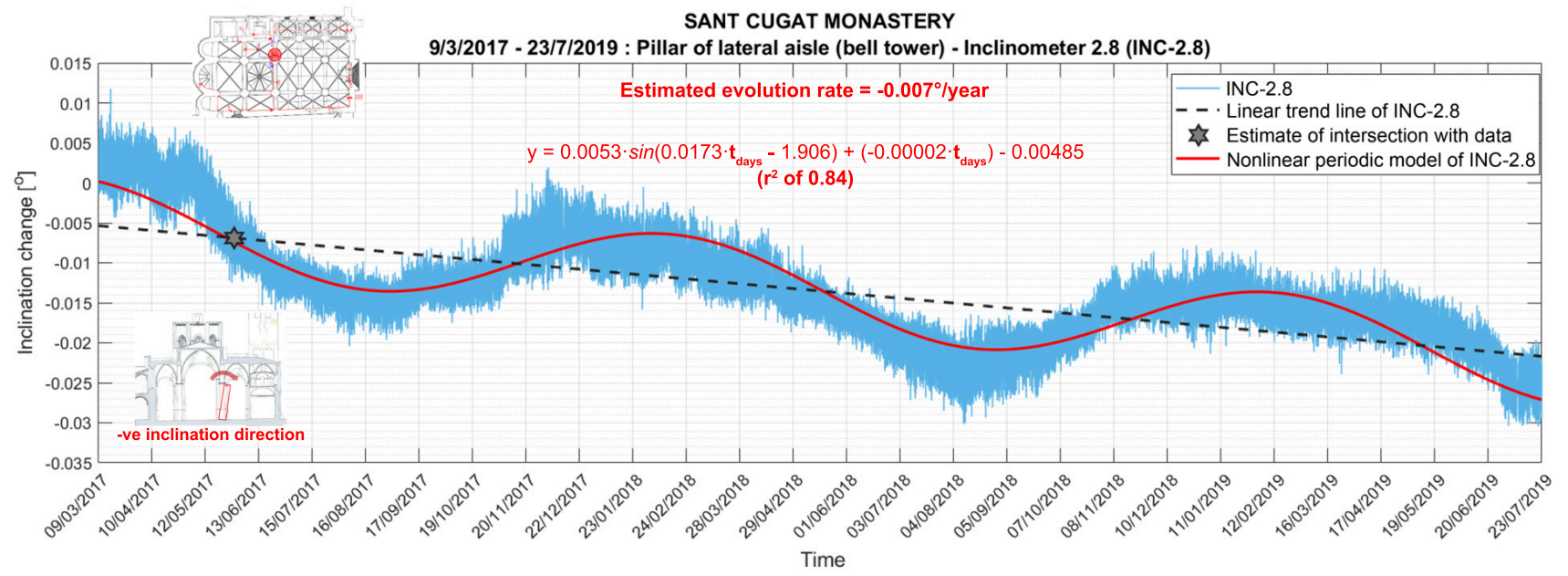

FIG URE 1 Linear and nonlinear periodic model fitted to data. An estimate of the first intersection point of the data with the fitted linear trend is shown in the figure.

However, it must be said that as with many nonlinear fitting algorithms, the Levenberg-Marquardt algorithm is susceptible to converging at a local minimum rather than a global one. Hence, particularly when dealing with large data sets acquired over long time periods, adequate convergence was found to rely heavily on setting appropriate initial estimates of the unknown parameters. In this case, a good initial estimate of the amplitude can be obtained directly from the data while the main period can be expected to relate to the duration of a tropical year (approximately 365.24 days ${ }^{44}$ ). Initial estimates of the remaining parameters can be obtained using results from the linear regression procedure. A summary of adequate initial estimates for all the unknown parameters in Equation (1) is given in Table 2.

As shown, the initial value of the coefficient related to phase shift relies on an estimate of the position of the first intersection point with the straight line fit. This was obtained by subtracting the identified linear trend from the raw data and identifying the first two points between which there was a change of sign. Linear interpolation between these two points provided an estimate of the time to the first intersection point. In order to avoid intersection points caused by higher frequency fluctuations not representative of the seasonal period, this procedure was carried out after removing higher frequency 
components from the data using a multi-level wavelet decomposition with Symlet Wavelets ${ }^{45 ; 46}$. An example of the estimated intersection point is shown in Figure 1.

TAB LE 2 Summary of initial estimates specified for each response variable for the nonlinear fitting procedure.

\begin{tabular}{|c|c|}
\hline $\begin{array}{l}\text { Parameter in } \\
\text { Eqn. (1) }\end{array}$ & Initial estimate \\
\hline A & $\begin{array}{c}A_{0}=\frac{\max \left(y_{i}\right)-\min \left(y_{i}\right)}{2}, \text { where } y_{i} \text { refers to the vector containing all the values of the response variable } \\
\text { recorded over the whole monitoring period. }\end{array}$ \\
\hline$P$ & $P_{0}=\frac{2 \pi}{365.242}$, if the $24 \mathrm{~h}$ day is used as the base unit of the duration vector. \\
\hline$\phi$ & $\phi_{0}=P_{0} \times$ Estimated position of the first intersection point of data with straight line fit (see Figure 1) \\
\hline B & $B_{0}=$ Slope of trendline identified from linear regression \\
\hline C & $C_{0}=y$-intercept of trendline identified from linear regression \\
\hline
\end{tabular}

A significant improvement of this method compared to linear regression lies in the fact that it attempts to model the actual nonlinear behaviour of the response variable. Selected metrics can thus be used to assess the quality of the fit and to reject trend estimates when the nonlinear periodic model cannot represent the evolution of the structural parameter. Two simple metrics were found to be particularly useful for this purpose. The first one being the well-known coefficient of determination $\left(r^{2}\right)^{47}$, which ranges from 0 to 1 , with a value closer to 1 indicating a better fit. Based on results from the case studies forming part of this research, it is recommended to dismiss evolution rates when the method yields a coefficient of determination lower than 0.6 . The other useful metric was found to be the percentage by which the final identified period differs from 365.24 days. The rationale being that since seasonal cycles rely on the movement of the sun, if the periodic component is to represent them, it should have a period close to the duration of a tropical year. As such, if the final identified period is found to differ by more than $25 \%$ from 365.24 , the estimated evolution trend should be rejected.

Despite this improvement, the method still fails to explicitly asses the effect that measured environmental parameters have on the measured structural parameters. As such, the estimates can be significantly biased by underlying trends or irregular changes in environmental parameters. Once again, this makes it difficult to decide which thresholds reflect an actual evolutionary or stationary condition.

\section{2 | Preliminary evaluation of correlation with monitored environmental parameters}

It is clear that variations of environmental parameters, notably changes in temperature, are the root cause of reversible seasonal changes experienced by most masonry structures. Since some of these parameters can easily be monitored, taking advantage of such measurements to filter-out their effect from the evolution of structural parameters can definitely provide an improvement on the method presented in Section 2.1.

Before attempting any procedure using actual measurements of environmental parameters, it is important to determine which ones have the greatest influence on the evolution of each response. This can be achieved by computing the Pearson correlation coefficient ${ }^{47}\left(R_{X, Y}\right)$ between measured environmental and structural parameters. This coefficient can be understood as a normalised version of covariance between two random variables and hence represents a dimensionless measure of their linear dependence. It can vary between -1 and +1 with absolute values closer to unity indicating a better correlation. The sign of the coefficient indicates the type of correlation. A negative sign means that an increase of one parameter leads to a decrease of the other while a positive one signals the opposite. Hence, following this preliminary evaluation, monitored environmental parameters showing the strongest influence on structural ones can be chosen for the subsequent analyses presented in the following sections.

\section{3 | Filtering environmental effects using linear regression with selected predictors}

One method which explicitly considers measured environmental parameters (predictors) relies on the assumption that their effect on responses can be represented by a perfectly linear model. The two unknown parameters of such a model can be identified through simple linear regression between recorded values 
of each response and the chosen predictor as shown in Figure 2. Due to the simplistic nature of the assumed relationship, it is clear that effects caused by structural mechanisms of interest can influence the identified linear models. Hence, one can argue that using data only from a single seasonal cycle for the regressions will provide the most suitable models. On the other hand, since the relationship between some predictors and responses change over several seasonal cycles, it can also be argued that data from the entire monitoring period can better represent their dependency. Hence, models are identified for both cases (listed below) and the results are then compared. In fact, the differences between them are used in the proposed classification procedure to evaluate the evolutionary state of monitored responses.

1. Linear filter (i): Linear regression between selected predictor and response variable using data from the entire monitoring period.

2. Linear filter (ii): Linear regression between selected predictor and response variable using data only from a full calendar year (this period will hereafter be referred to as the estimation phase).

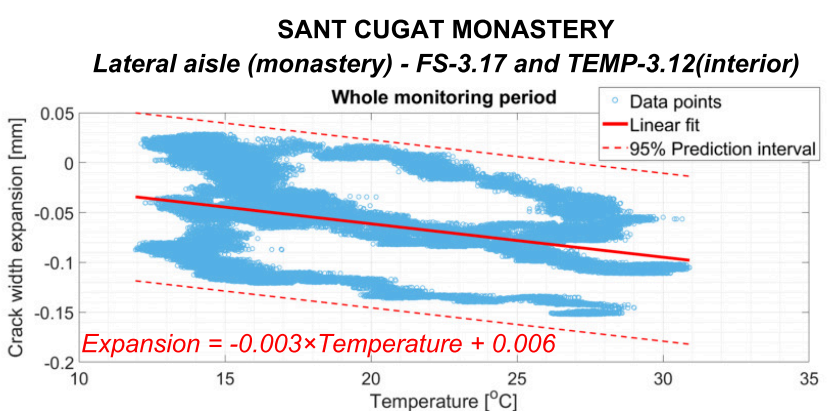

(a)

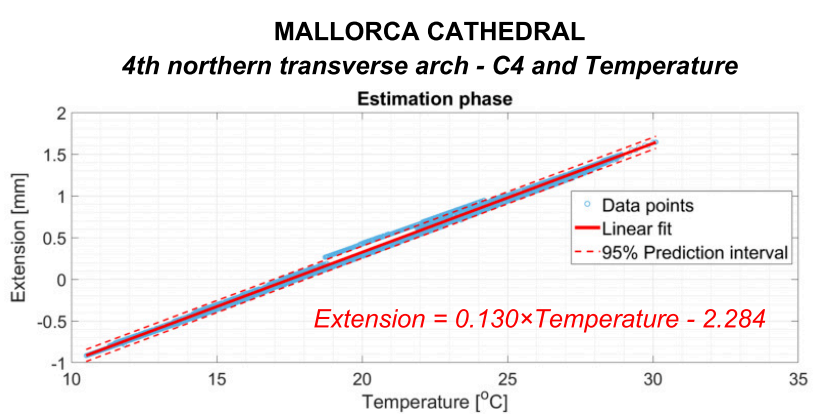

(b)

FIGURE 2 (a)Example of linear regression between crack width expansion and temperature over whole monitoring period. (b)Example of linear regression between the monitored span of an arch and temperature over an estimation phase of one year.

Following the regression procedure, measured values of each selected predictor are substituted into the corresponding linear model to simulate changes of the structural parameter caused by the environmental one. Since variations experienced by structural parameters can be considered as the result of a series of phenomena, the actual measurements of the latter are then filtered by simply subtracting the simulated effect, as shown in Figure 3. Estimates of the underlying annual evolution rates $\left(E R_{\text {lin(i) }}\right.$ and $\left.E R_{\text {lin(ii) }}\right)$ are then obtained by carrying out simple regressions of these filtered residuals.

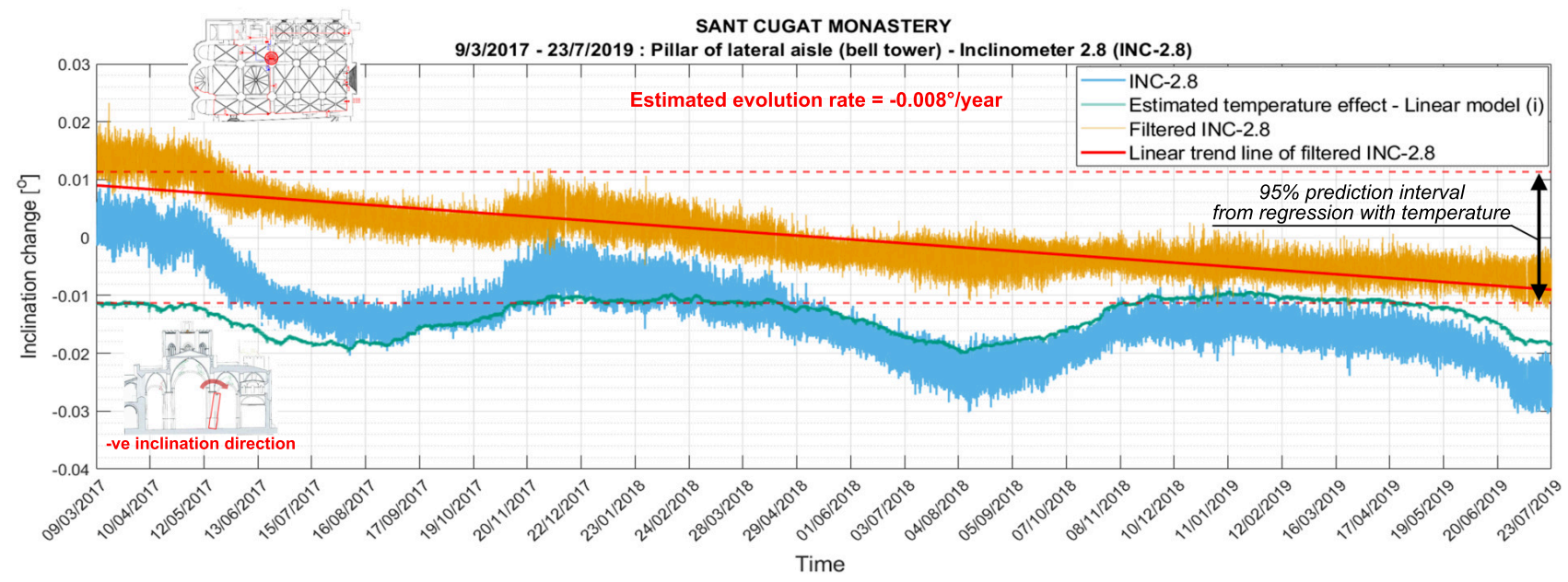

FIGURE 3 Filtering of temperature effect based on identified general linear trend. (For interpretation of the references to colour in this figure, the reader is referred to the web version of this article)

A significant advantage of this method when compared to the previous two is that it explicitly uses measured predictors and hence allows an assessment of how well each linear model can predict the relationship between environmental and structural parameters using common error metrics. In fact, if the residuals are assumed to be normally distributed when no significant structural mechanism is present, a prediction interval representing a specific level of confidence can be obtained based on the dispersion of residuals. This can prove extremely useful for the interpretation of results since it is expressed in the 
same units as the monitored structural parameter.

\section{3 | PROPOSED ANALYSIS METHODOLOGY}

Although it is clear that simple linear models can provide a good representation of the relationship between environmental and structural parameters (Figure 2(b)), in many cases, it fails to represent the relationship adequately (Figure 2(a)). One of the main reasons for this is the fact that the simplistic nature of the model cannot take into consideration certain effects influencing the system it aims to describe, such as those due to thermal inertia.

Hence the proposed automated data analysis methodology developed as part of this research consists of two parts. The first part aims to improve upon the method presented in Section 2.3 by exploiting dynamic regression models to describe the relationship between selected predictors and structural parameters more reliably. The process can be applied to a complete SHM system and includes a procedure for estimating model orders that are best suited for system identification, as well as provisions to deal with irregularly sampled and missing data.

The second part of the methodology aims to facilitate the interpretation of the predicted evolution rates after the filtering process. This is achieved by classifying the state of each monitored structural parameter based on the evaluated underlying trend and the level of uncertainty associated to the models describing its relationship with selected environmental parameters. Since the linear models described in Section 2.3 are clearly adequate for certain cases, the classification procedure also takes advantage of predictions from this method. Hence, a preliminary step to the proposed data analysis procedure involves carrying out all the analysis methods presented in Section 2.

\subsection{Filtering environmental effects using dynamic statistical models (ARX models)}

The procedure of utilising ARX models for evaluating any underlying trends present in response variables involves selecting suitable model orders by adopting some quality criteria. The coefficients of the ARX models can then be estimated using QR factorization ${ }^{48}$ based on measurements collected during an estimation phase. In order for the model to capture most of the reversible components caused by environmental effects during a complete seasonal cycle, the estimation phase should ideally span at least 1 full year. Once this model has been estimated and validated, it can be used together with data collected after the estimation phase to simulate responses based on measured predictors. The residuals obtained by subtracting the simulated behaviour from its recorded counterpart then allows changes related to irreversible structural damage to be distinguished from reversible ones caused by varying environmental conditions.

As its name suggests, an Auto-Regressive with eXogenous input (ARX) model utilises measured values of past outputs together with those of past and current or delayed predictors to describe the dynamics of a system. The single-input single-output (SISO) form of the ARX model has the following structure:

$$
y \hat{(t)}+a_{1} y(t-1)+\ldots+a_{n a} y(t-n a)=b_{1} x^{e n v}(t-n k)+\ldots+b_{n b} x^{e n v}(t-n b-n k+1)+e(t)
$$

Where $y(t)$ is the predicted response at time $t, e(t)$ is the white-noise disturbance value, $n a$ and $n b$ are the auto-regressive and exogenous orders of the ARX model, and $n k$ is the delay. Specifically, na and $n b$ refer respectively to the number of past samples of the response and predictor variables used for identification and $n k$ is the number of samples of the predictor variable that occur before the predictor starts influencing the response. Hence $y(t-1) \ldots y(t-n a)$ refer to the previous responses on which the current one depends, while $x^{\text {env }}(t-n k) \ldots x^{\text {env }}(t-n b-n k+1)$ refer to the previous and delayed predictors on which the current response depends. The multiple-input single-output (MISO) form of an ARX model has the same structure as that described by Equation (2) but with additional parameters to incorporate any number of additional predictors. Therefore, for each new predictor incorporated in the model, a new exogenous order and delay has to be specified.

The first step of the filtering procedure involves selecting which measured environmental variables will be used as predictors for the representation of the dynamic system as an ARX model. Two possible 
candidates that are commonly monitored as part of SHM systems are temperature and relative humidity. It can be expected that temperature will have the greatest influence on the evolution of the response variable. Since relative humidity measurements were available in addition to temperature ones for one of the case studies forming part of this research, the results from the preliminary evaluation of correlation described in Section 2.2 were compared. Indeed, the results reveal that for every monitored structural parameter, the linear correlation is stronger with temperature than with relative humidity. In fact, for almost every response investigated as part of the two case studies, the dependency on temperature is clearly visible when examining daily fluctuations. Hence, SISO ARX models with temperature as the sole predictor constitute the most basic ARX model that should be identified for every monitored response. If temperature is recorded at more than one location (for instance in the interior and at the exterior of the structure), the data from the temperature sensor showing the greatest Pearson correlation coefficient with the response should be used as the predictor in the SISO ARX model.

Recent years have been marked by a significant increase in the ability of modern computers to exploit large amounts of data for system identification. As such, in addition to the SISO ARX models described in the previous paragraph, the proposed data analysis methodology also incorporates the use of MISO ARX models to filter out seasonal variations when multiple suitable environmental parameters are monitored. Naturally, the decision of which environmental parameters to include in the models has to be addressed. It is undeniable that the presence of moisture in the masonry fabric can significantly alter its mechanical behaviour ${ }^{49-51}$ and hence influence the response of structures. However, the design of an adequate acquisition protocol for the monitoring of parameters representative of the water absorption phenomena can be a challenging task, particularly for large complex masonry structures ${ }^{52}$. Some authors have attempted to include measured relative humidity on-site as a predictor in a MISO ARX model in an attempt to filter out moisture related effects from natural frequencies being monitored through a dynamic monitoring system ${ }^{38}$. Although it was clear that water absorption had a notable effect in this case due to observed changes of the natural frequencies at the beginning of rainy seasons, the inclusion of this predictor provided no improvement in the model's ability to describe the environmental variability. This can be attributed to the fact that measurements made by humidity sensors on the exterior of walls actually reflect the level of moisture in the air and not in the masonry. For this reason, the proposed methodology does not include MISO ARX models with relative humidity as a predictor, even if it is good practice to monitor it in most SHM systems. However, it was envisaged that temperature gradients between interior and exterior temperature could significantly influence the dynamics of the system. Since both are often recorded as part of modern SHM systems, it is recommended to utilise MISO ARX models that incorporate both as predictors to characterise the environmental influence on the evolution of monitored structural responses. Nevertheless, the proposed methodology can be extended to include any monitored environmental parameters causing reversible variations of structural responses such as the moisture content in the masonry or the level of water in the foundation soil. The latter can be monitored with piezometers and the former could possibly be monitored by humidity sensors placed at different locations both along the thickness of masonry walls and at different levels of the structure ${ }^{53}$. However, it should be noted that in some cases, particularly for masonry structures with an interior leaf made of lime concrete, moisture diffusion can produce a redistribution of stresses over several centuries ${ }^{54}$ which can even be the root cause of a slow irreversible deterioration mechanism. Similarly, changes in water table levels can sometimes cause significant differential settlements over long time periods resulting in the activation of a progressive damage mechanism ${ }^{55 ; 56}$. Under such circumstances, it would be undesirable to include these monitored parameters as predictors in the MISO ARX model at the filtering stage since the trends they induce can be essential for an accurate diagnosis of the structure.

Once predictors to be used in the ARX models have been selected, the next step of the procedure involves extracting data from the estimation phase. This represents the data set for which the errors between the model output and the measured response will be minimised. In most cases it is perfectly adequate to take data collected during the first complete year as the estimation data. However, if there are significant periods for which data is missing during the first year of monitoring, it is recommended to consider any period lasting one year which has the least amount of missing data as the estimation phase.

It is key to adequately condition the estimation phase data before carrying out any system identification. Besides converting raw signals from sensors to meaningful physical units and removing clear anomalies, another procedure which can be useful to implement involves resampling the data. This is 
particularly useful to reduce the computational cost of system identification in cases for which the data is sampled at a high rate. Since the damage phenomena of interest typically progresses slowly over many years, if the SHM system samples data at intervals shorter than an hour, noticeable savings in terms of computational cost can be achieved by increasing the sampling interval to an hour without any significant loss of accuracy in terms of the predicted evolution rates. Naturally, after using the ARX models to simulate the environmental effect, the data will have to be upsampled back to the original sampling rate so that the residuals can be computed from the measured values of the response variables in the same way they were for the method described in Section 2.3. Upsampling is a lossless procedure and hence straightforward application of spline interpolation ${ }^{57}$ can safely be used for this purpose. However, for the downsampling procedure, great care has to be taken to prevent aliasing and avoid distorting the original signal excessively. To prevent aliasing, a lowpass anti-aliasing filter should be implemented before the resampling procedure. The question then arises as to which interpolation method will prove to be both robust and accurate in representing the original signal. Three different methods were tested as part of this research: Linear interpolation using 2 neighbouring points as well as piecewise cubic ${ }^{58}$ and spline interpolation ${ }^{57}$, both of which use 4 neighbouring points. Linear interpolation resulted in the smallest maximum and mean errors for data from all the 16 sensors tested. In every case, the mean error was several orders of magnitude smaller than the minimum resolution of the sensor and the maximum error smaller than the greatest daily variation experienced by the sensor. As such, it was concluded that using linear interpolation for downsampling is most adequate. Although most modern data loggers will accurately provide data at a uniformly sampled rate, analysing data collected from more archaic systems could create a need for system identification from irregularly sampled data. Since such data is not easily handled by discrete-time model identification techniques ${ }^{59}$, it is recommended to resample the data to a uniform rate using the same procedure described in this paragraph before carrying out system identification in such cases.

A particular characteristic of ARX models is that the equation error is modelled as being a zero-mean white process with unknown variance ${ }^{60}$. In fact, the noise model is coupled to the identified parameters of the response variable and hence the only unknown parameter related to noise that needs to be solved for during the identification process is the variance of the noise term. Although this means that the noise and the dynamics of the system cannot be modelled independently, the simplicity of the scheme creates no stability problems in optimal predictors and allows unbiased estimates of the parameters by means of least squares. However, it is crucial to remove the mean from both the response and predictor data before carrying out system identification to avoid an offset term in Equation (2). In fact, it is good practice to normalise the input and output data for this system identification task ${ }^{41}$. Therefore, for each estimation data set, the result after removing the mean should also be divided by the standard deviation. It is important to store both the means and the standard deviations, since it is instrumental to transform all the identified ARX models back to the engineering units of the original data.

Before carrying out system identification using the normalised data sets, a choice needs to be made on the delay to be specified. If the environmental and structural parameters are acquired simultaneously, it is perfectly adequate to simply assume a delay of 0 in most cases. This is particularly true when the environmental parameter is temperature, as is the case for the recommended ARX models for the static SHM case. However, in some cases, the model could benefit from a short dead time occurring before the first predictor it uses to simulate the current response. In order to evaluate the most suitable delays for each response, the proposed procedure includes a quick delay estimation computation based on the comparison of ARX models with orders of 8 , evaluated for different delays spanning from 0 to 48 hours. As can be expected, out of the 44 ARX models estimated for the case studies (28 SISO and 16 MISO models), the delay estimation computation suggested that a delay of 0 was most appropriate for most of the cases. Moreover, only delays of up to 12 hours were suggested for the 12 cases for which it was deemed that a dead time would be suitable.

The last step remaining before estimating the parameters of the ARX models involves specifying appropriate auto-regressive and exogenous model orders. In essence, these control the duration in the past that is considered by each model to predict responses since it defines the number of past samples used for prediction. Choosing the right combination of model orders is no straightforward task but this can greatly influence the quality of the final models. For instance, it is likely that responses being significantly affected by a structural mechanism would benefit more from a higher auto-regressive order while those influenced 
by complex environmental phenomena would benefit more from higher exogenous model orders. Hence, a systematic procedure was developed as part of this research to select suitable combinations of model orders for each response from a pre-defined range. The procedure involves dividing the estimation data of each response equally to form an estimation and a validation subset. Models for each of the structures defined by the pre-defined range are then estimated using the estimation subset. The loss functions are then computed for these models when applied to the validation subset. The loss function $(V)$ refers to the error that is minimised by the least-squares method during system identification. As shown by Equation (3), it can be defined as the normalized sum of squared prediction errors.

$$
V=\frac{1}{N} \sum_{k=1}^{N} e_{k}^{2}
$$

Where $N$ is the number of measurements, $k$ is the time step and $e_{k}$ are prediction errors defined as the measured response minus the predicted one.

Following the computation of loss functions, the structure resulting in the lowest error when applied to the validation data set is specified for each response. The parameters of these models with known structures can then be estimated using all the data from the estimation phase. A significant benefit of the order selection procedure is that it relies on loss functions computed on validation subsets and hence the final ARX models already inherently include a process that helps ensure that they are useful not only for the estimation data, but also for new data from subsequent phases of the monitoring period. However, the task of defining the range of model orders that need to be tested still needs to be addressed. Since it was expected that one of the main benefits stemming from the dynamic nature of ARX models would be their ability to consider thermal inertia effects, an investigation was carried out on the required model orders that would theoretically be able to encompass most of the effects of this physical phenomena. In general, the thermal inertia of a building envelope causes two noticeable differences between external and internal temperature fluctuations: a decrement of the amplitude and a time lag between the two ${ }^{61}$. Previous research reveals that the duration of these thermal lags rely heavily on the thermophysical properties of wall elements and that lags of approximately 12 hours can be observed from experiments on brick masonry walls with a thickness of less than $30 \mathrm{~cm}^{62}$. It can be expected that thermal inertia effects will be substantially different in masonry heritage structures as they can be characterised by massive external walls that are often thicker than $1 \mathrm{~m}$. Based on measurements from one of the case studies, most of the time lags between external and internal temperature appear to last less than a day. However, in some extreme cases, time lags lasting several days could be identified. Based on these observations, the ranges listed in Table 3 were tested as part of this research. If the acquisition was made at a higher rate, the estimation data was resampled to a sampling period of 1 hour.

TAB LE 3 Ranges of ARX model orders tested using estimation data with a sampling period of 1 hour.

\begin{tabular}{|c|c|c|c|cc}
\hline Corresponding duration [days] & \multicolumn{2}{|c|}{ SISO ARX - model orders tested } & \multicolumn{2}{c|}{ MISO ARX - model orders tested } \\
\hline Lower limit & Upper limit & Lower limit & Upper limit & Lower limit & Upper limit \\
\hline 1 & 4 & 24 & 96 & 24 & 96 \\
\hline 4 & 6 & 96 & 144 & 96 & 144 \\
\hline 8 & 10 & 192 & 240 & 192 & 240 \\
\hline 4 & 10 & 96 & 240 & 96 & 240 \\
\hline 4 & 15 & 96 & 360 & - & - \\
\hline 5 & 20 & 120 & 480 & - & - \\
\hline 5 & 25 & 120 & 600 & - & - \\
\hline
\end{tabular}

The results from all the ranges tested reveal that it is important to specify a lower limit in the range to prevent the model structure determined by the order selection procedure from being overly dependent on only past responses or predictors. A lower limit corresponding to at least four days is recommended. It was also found that specifying a larger range leads to more accurate final models even if the upper limit of the range is unchanged. For instance, specifying a pre-defined range of 4 to 10 days yielded more accurate models than specifying one of 8 to 10 days. Of course, the final range selected is largely 
dependent on the computational expense that can be spared but the maximum ranges listed in Table 3 are recommended for SISO models and MISO ones with 2 predictors.

Once the final models have been identified for each monitored response, they can be used to simulate the expected responses from new measurements of predictors. It is very important to highlight the distinction made between simulation and prediction in the realm of system identification since one of the greatest advantages of using ARX models for the analysis of static SHM systems stems from this difference. It is clear from Equation (2) that both past responses and predictors are used to describe the dynamics of the system. The data from both are therefore used during the estimation phase. However, there are two ways of generating a model response: it can be predicted or simulated. Prediction involves computing the model response at a particular point in time using values of measured predictors and past responses. Simulation on the other hand, involves computing the model response using only measured predictors. Effectively, this means that although the model is able to account for changes that are likely due to structural mechanisms through the parameters associated to past responses, it is also able to exclude those when computing the expected response by only using terms of the model that correspond to predictors. This is ideal for the case of static SHM since it is often impossible to isolate a period of time during which the relationship between environmental predictors and structural responses can be considered as being completely isolated and free from effects caused by active structural mechanisms. Any evolution trend is then estimated by carrying out a regression of the residuals obtained by subtracting the simulated environmental effect of the responses from their measured values.

Two different goodness-of-fit measures were used to compare the effectiveness of the linear, SISO ARX and MISO ARX models in representing the relationship between predictors and responses: the previously described coefficient of determination $\left(r^{2}\right)$ and 1.96 times the standard error of the estimate ${ }^{63}$ computed from the residuals $\left(1.96 \sigma_{e}\right)$. The latter can be interpreted as a typical distance of measured data points from model predictions. It was chosen because it is used to define the $95 \%$ prediction interval from a normally distributed sample and because it is in the same units as the responses. In all cases, these were computed from the measured and simulated values of responses over the time period used to estimate the model. As will be seen in Section 4, for almost all of the 28 responses monitored as part of the two case studies, the ARX models were able to represent the environmental variation much more accurately than the linear models.

\section{2 | Interpretation of results}

As a result of the analyses presented in Sections 2.3 and 3.1, an estimated filtered evolution rate is obtained for each response variable. In fact, since these methods aim to directly model the relationship between environmental and structural parameters, the standard error of the estimate computed from residuals for each model over the estimation phase represents a certain level of confidence in the model's ability to predict variations of the response. Since such metrics are in the same units as the response, they can be compared directly to evaluate the level of certainty associated to the estimated filtered evolution rates. In order to provide a systematic way of doing so, the current research proposes a multi-step classification approach based on comparing results obtained from the methods presented in Sections 2.3 and 3.1 through a series of 5 logical tests (see Figure 5). Two key values obtained from the most extensive ARX-based procedure used are employed in every test: the filtered annual evolution rate $\left(E R_{A R X}\right)$ and 1.96 multiplied by the standard error of the estimate computed over the estimation phase $\left(1.96 \sigma_{e-A R X}\right)$. The approach can be programmed so that in addition to the predicted rates, a label is automatically assigned to each monitored response. Based on the outcomes of the tests, each response is classified in one of the following four categories:

1. Stationary: Monitored parameters showing a clear stationary trend outside reversible variations caused by environmental parameters.

2. Evolutionary: Monitored parameters showing a clear evolutionary trend outside reversible variations caused by environmental parameters.

3. Apparently stationary: Monitored parameters showing a stationary trend but for which there still is a rather large uncertainty associated to the estimation of the trend.

4. Apparently evolutionary: Monitored parameters showing an evolutionary trend but for which there 
still is a rather large uncertainty associated to the estimation of the trend.

5. Inconclusive: Monitored parameters for which no clear conclusion can be made on its evolutionary state from the available monitoring data alone.

The first test of the classification procedure aims to identify responses which clearly show a stationary trend. Since classifying a response as stationary when it is experiencing an active trend can have dangerous consequences, relatively strict conditions have been established for this test. Specifically, $1.96 \sigma_{e-A R X}$ must be lower than 5 times the minimum effective resolution of the sensors and $2 E R_{A R X}$ (the estimated evolution over two years) must be lower than 0.005 units. In addition to these two requirements, this test includes another requirement based on the normality of the residuals. This stems from the understanding of a truly stationary time-series as one that can be represented by a model with Gaussian errors ${ }^{34}$. Hence, it can be said that if the residuals after the filtering process are normally distributed, the model has been able to capture most of the environmental variability, and the response does not contain any underlying trend caused by active structural mechanisms. Naturally, in order for this requirement to be incorporated in an automated procedure, one has to quantify the degree of normality of the residuals. There exist many different tests to verify if observations are from a normal population and most of them rely on computing a test statistic and a critical value. The latter is usually dependent on the sample size and a chosen significance level. The hypothesis that the sample belongs to a normal population is then rejected or accepted based on the relationship between the test statistic and the critical value. In the case of data from static SHM, the sample size is usually very large since it contains data sampled every hour over several years. Moreover, the dynamics of the system are often very complex making it difficult for any model to eliminate all reversible effects perfectly. As such, the sample set containing residuals will almost always fail to satisfy most established normality tests irrespective of the chosen significance level. Nevertheless, it is clear that in some cases, the residuals can be well represented by a normal distribution (see Figure 4).

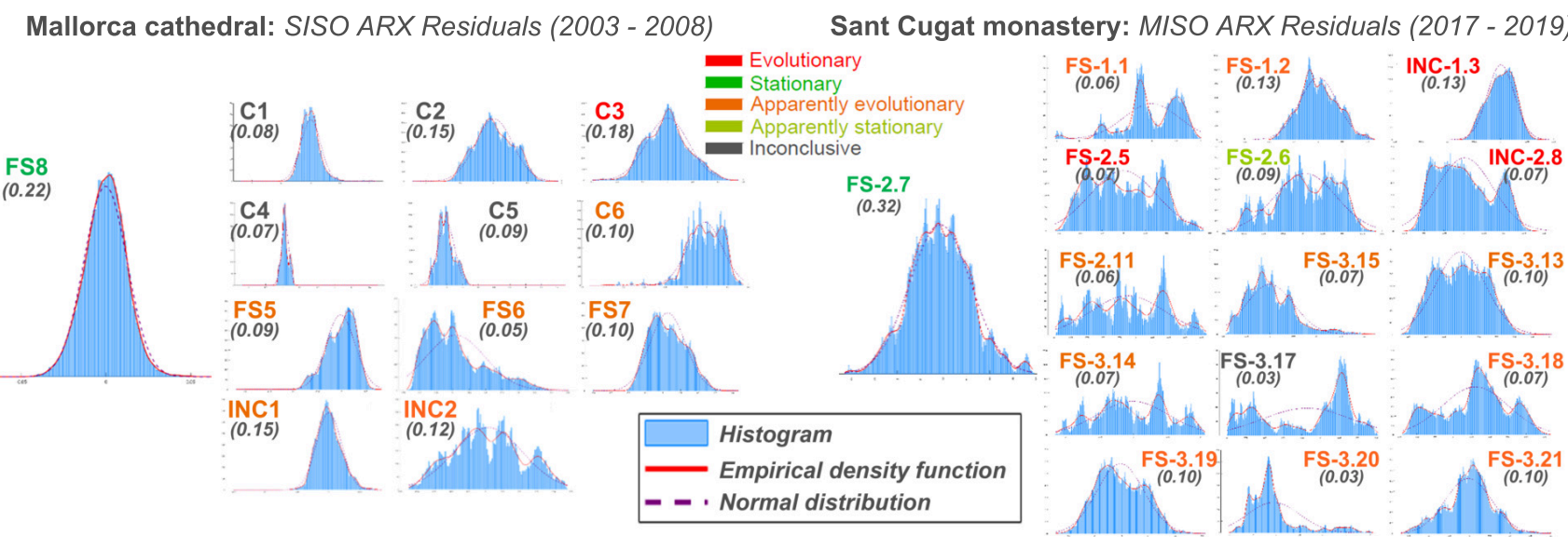

FIG URE 4 Empirical distribution of ARX Residuals for responses forming part of case studies. Values of the adapted Lilliefors ratio for the case of static SHM is also shown. The colour of the sensor name relates to the final estimated condition according to the proposed classification procedure. (For interpretation of the references to colour in this figure, the reader is referred to the web version of this article)

Hence, a modified normality test based on the Lilliefors Test ${ }^{64}$ was developed for this purpose. As is the case for the original test, the test-statistic (kstat) is computed by finding the maximum deviation of the empirical cumulative distribution function (CDF) from the CDF of a normal distribution with the same mean and standard deviation as the sample data set. The original test rejects the hypothesis of normality if the test statistic is greater than the critical value. Hence the sample is said to be from a normal distribution if the ratio of the critical value over the test statistic is greater than or equal to 1 . In contrast to the original test, the modified one proposes a fixed critical value of 0.005 and requires the computation of an adapted Lilliefors ratio equivalent to $\frac{0.005}{\text { kstat }}$. These ratios computed for the residuals obtained from the most extensive ARX-based procedures applied to the responses forming part of the case studies, are shown in Figure 4. Based on these observed ratios, it is suggested that for the case of static SHM, residuals that have an adapted Lilliefors ratio greater than 0.2 can be considered as being 
normally distributed. Hence, responses that satisfy this requirement together with the first two presented at the start of this paragraph can be considered as being stationary.

The second test aims to identify responses that clearly show an evolutionary trend. Hence, the estimated evolution rate should be significant in comparison to the error associated to modelling the environmental variation and this error should be sufficiently small. It must be noted that in certain cases, if a structural parameter is particularly responsive to changes in environmental conditions, it can experience large reversible variations. In such cases, even if the ARX model is able to accurately simulate the seasonal variations, the value of $1.96 \sigma_{e-A R X}$ might still be significant in comparison to the minimum effective resolution of the sensor. In order to avoid penalising the accuracy of the model in such cases, a small value of $1.96 \sigma_{e-A R X}$ was defined as the maximum between 5 times the minimum effective resolution of the sensor and $20 \%$ of the average maximum daily variation experienced by all sensors in the SHM system measuring the same type of response. If the residuals satisfy this requirement and $2 E R_{A R X}$ is greater than $1.96 \sigma_{e-A R X}$, the response is classified as evolutionary.

The subsequent tests are not as rigorous as the first two but intend to utilise most of the useful information obtained from the various proposed analysis procedures to provide an informed estimate of the condition of each structural response being monitored. The third test classifies a response as apparently stationary if $1.96 \sigma_{e-A R X}$ is less than 0.05 and $2 E R_{A R X}$ is less than $0.4 \times 1.96 \sigma_{e-A R X}$. Test 4 relies on three requirements to classify a response as apparently evolutionary: $1.96 \sigma_{e-A R X}$ must be less than $0.2,2 E R_{A R X}$ must be greater than $0.4 \times 1.96 \sigma_{e-A R X}$ and the Pearson correlation coefficient computed between the response and relevant temperature records must be greater than 0.6 . The rationale being that a response is most likely evolutionary if its relationship with temperature can be well represented by a linear approximation and the predicted trend after filtering out simulated environmental effects is significant in relation to the error associated to this simulation. The final test relies on a comparison between the estimated evolution rates from all methods attempting to directly filter out reversible effects caused by measured predictors $\left(E R_{\operatorname{lin}(i)}, E R_{\operatorname{lin}(i i)}, E R_{\text {SISO-ARX }}\right.$ and if applicable $\left.E R_{\text {MISO-ARX }}\right)$. The test classifies the response as apparently evolutionary if the estimated rates from all these methods agree to within $25 \%$ (normalised to the lowest evolution rate for each response) and if $2 E R_{A R X}$ is greater than $0.45 \times 1.96 \sigma_{e-A R X}$. A greater requirement is imposed on the evolution rate in comparison to Test 4 because there is less physical meaning associated to this test. Although a response can satisfy both Test 4 and Test 5 , Test 3 has been designed to be mutually exclusive from these two.

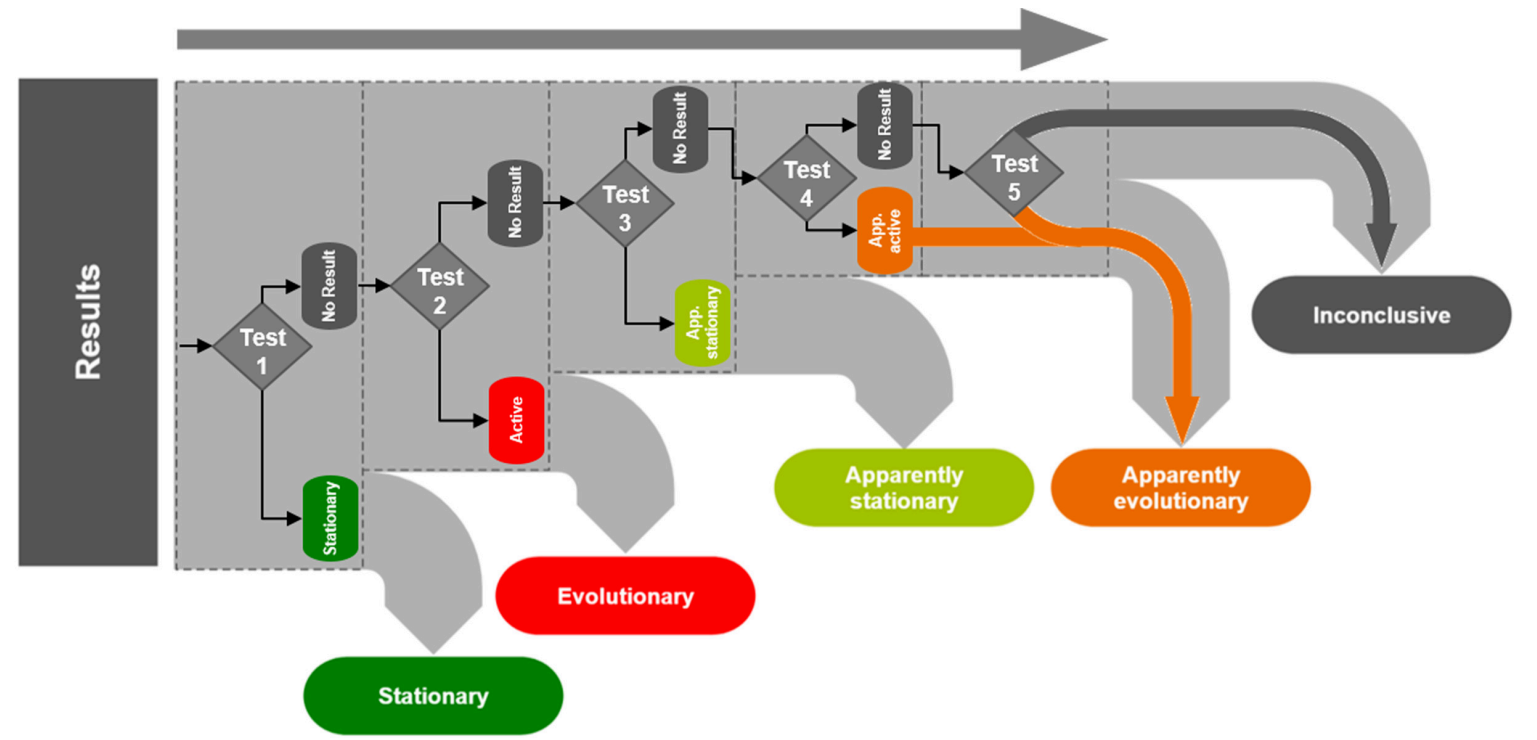

FIGURE 5 Procedure for interpretation of results after filtering effect of environmental variability.

The overall classification procedure is summarised in Figure 5. As can be seen, if Tests 1, 2 or 3 are satisfied, the results related to that particular response does not undergo any further tests since it would already have been assigned a label. If a response fails all tests, it is classified as inconclusive. One of the main advantages of the classification procedure is that it can greatly facilitate how the estimated evolution rates should be prioritised during the prognosis. Basically, the possible underlying mechanisms related to responses classified as "Evolutionary" should be investigated first followed by those labelled 
as "Apparently evolutionary" that show the greatest ratio between $E R_{A R X}$ and $1.96 \sigma_{e-A R X}$. Responses labelled as "Inconclusive" often require an extended period of monitoring in order to shed more light on their actual state. Of course, longer monitoring periods will also help develop a greater level of certainty on any diagnosis made from the data analysis, particularly if the cause of some estimated trends cannot be explained.

\section{4 | CASE STUDIES}

The two case studies forming part of this research consist of medieval churches and cathedrals. Such structures were usually built over very long time periods and still represent some of the most daring and complex examples of masonry design. The first case study, the cathedral of Santa Maria in Palma de Mallorca, was built progressively from the apse to the façade over a period of 300 years, from 1306 to $1600^{65}$. The cathedral boasts grand proportions and presents many structurally audacious aspects. The most daring of which probably is the slenderness of the columns ${ }^{2}$, reaching a ratio of 14.2 in some areas while the value encountered in other Gothic cathedrals usually ranges from 7 to $9^{66}$. For all these reasons, it represents one of the most emblematic monuments of the Catalan Gothic Style. The second case study, the monastery of Sant Cugat, is located in Sant Cugat del Vallès, Catalonia. The monastery is composed of a cloister and a church, with the latter being the main focus of the study. The masonry structure at the site today consists of various parts built over different time-periods, mostly from the mid-12th century to the 15th century. The interaction between different parts results in a complex overall structural behaviour, adding to the difficulty of the diagnosis.

\section{1 | Mallorca cathedral}

\subsection{1 | SHM system and results}

A five-year monitoring system was installed in the cathedral in 2003 to better understand the complex behaviour of the structure and to identify any active mechanisms possibly contributing to its deterioration. In addition to temperature and humidity sensors, the system consisted of 6 convergence extensometers monitoring changes in the distance between two points, four crackmeters monitoring changes in crack widths and two inclinometers monitoring changes of inclination of key elements. The convergence extensometers and crackmeters had a resolution of $0.01 \mathrm{~mm}$ while the inclinometers had an effective resolution of $0.001^{\circ}$. Table 4 describes the location of every sensor monitoring a structural parameter and the total duration of useful data collected by each one over the five-year monitoring period. The predicted evolution rates from methods based on directly fitting time series to selected models are also shown in Table 4. The location of the sensors together with the final estimated evolutionary condition are also shown in Figure 7.

TA B LE 4 Summary of structural sensors used in the SHM system installed in Mallorca cathedral and estimated evolution rates from methods based on directly fitting time series to selected models.

\begin{tabular}{|c|c|c|c|c|c|c|}
\hline \multirow{2}{*}{ Sensor } & \multirow{2}{*}{ Location } & \multirow{2}{*}{ Years of data } & \multirow{2}{*}{ Type } & \multirow{2}{*}{ Units } & \multicolumn{2}{|c|}{ Evolution rate [unit/year] } \\
\hline & & & & & Linear regression & Nonlinear regression \\
\hline C1 & 4th central transverse arch & 1.2 & Extensometer & $\mathrm{mm}$ & 0.17 & - \\
\hline $\mathrm{C} 2$ & 4th central transverse arch & 4.9 & Extensometer & $\mathrm{mm}$ & 0.05 & - \\
\hline C3 & 4th southern transverse arch & 4.8 & Extensometer & $\mathrm{mm}$ & 0.10 & 0.09 \\
\hline C4 & 4th northern transverse arch & 3.7 & Extensometer & $\mathrm{mm}$ & 0.12 & 0.06 \\
\hline $\mathrm{C} 5$ & 8th southern longitudinal arch & 2.8 & Extensometer & $\mathrm{mm}$ & 0.02 & 0.05 \\
\hline C6 & 8th northern longitudinal arch & 4.2 & Extensometer & $\mathrm{mm}$ & 0.07 & 0.06 \\
\hline FS5 & Southern wall - 8th bay & 4.2 & Crackmeter & $\mathrm{mm}$ & 0.01 & 0.01 \\
\hline FS6 & Southern wall - 8th bay & 4.2 & Crackmeter & $\mathrm{mm}$ & -0.05 & -0.04 \\
\hline FS7 & Central nave - 6th bay & 4.2 & Crackmeter & $\mathrm{mm}$ & 0.06 & 0.08 \\
\hline FS8 & Central nave - 7th bay & 4.2 & Crackmeter & $\mathrm{mm}$ & 0.00 & 0.00 \\
\hline INC1 & 4th pillar (central nave - south) & 2.3 & Inclinometer & $\circ$ & 0.000 & 0.013 \\
\hline INC2 & Interior main façade & 1.9 & Inclinometer & $\circ$ & 0.036 & 0.034 \\
\hline
\end{tabular}


The evolution rate predicted for convergence extensometer $\mathrm{C} 1$ from the periodic model was disregarded because the identified period varied by more than $45 \%$ from the duration of a tropical year. On the other hand, that of convergence extensometer C2 was disregarded because it had a very poor correlation with the data (coefficient of determination of 0.19) indicating that the evolution of the data could clearly not be well represented by the nonlinear periodic model. Despite the greater sophistication of the nonlinear model, it can be seen that the estimated evolution rates between these two methods are in good agreement for certain cases, notably for convergence extensometers C3 and C6, for crackmeter FS5 and for inclinometer INC2.

Before discussing the estimated evolution rates predicted by the methods presented in Sections 2.3 and 3.1, a comparison of how well the linear and ARX models are able to represent the relationship between responses and predictors is presented in Figure 6. It is clear that the ARX models are better suited to represent the environmental variation in almost every case.

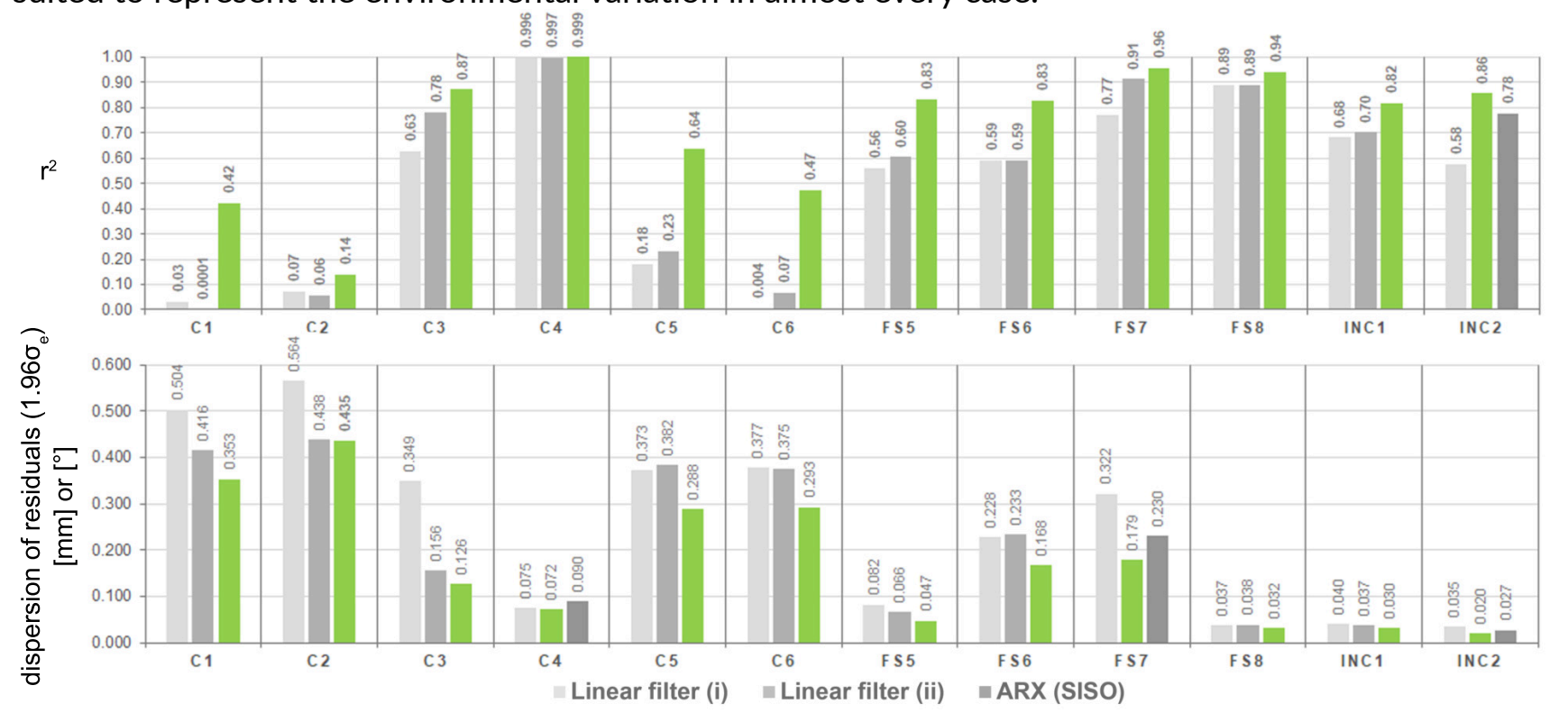

FIGURE 6 Coefficient of determination $\left(r^{2}\right)$ and dispersion of residuals $\left(1.96 \sigma_{e}\right)$ between simulated and measured responses in Mallorca cathedral.

The evolution rates estimated after filtering the simulated environmental effect using linear and ARX models are presented in Table 5. The estimated condition of each response using the procedure described in Section 3.2 is also shown in the table and illustrated in Figure 7.

TAB LE 5 Comparison of estimated evolution rates for monitored structural parameters of Mallorca cathedral from methods filtering out simulated effect of measured environmental parameters.

\begin{tabular}{|cc|c|c|c|c|}
\multirow{2}{*}{ Sensor } & \multirow{2}{*}{ Units } & \multicolumn{3}{|c|}{ Estimate of annual evolution rate [unit/year] } & \multirow{2}{*}{ Estimated condition } \\
\hline C1 & $\mathrm{mm}$ & 0.123 & 0.163 & 0.056 & Inconclusive \\
C2 & $\mathrm{mm}$ & 0.055 & 0.054 & 0.057 & Inconclusive \\
\hline C3 & $\mathrm{mm}$ & 0.081 & 0.088 & 0.077 & Evolutionary \\
C4 & $\mathrm{mm}$ & 0.017 & 0.016 & 0.002 & Inconclusive \\
\hline C5 & $\mathrm{mm}$ & 0.007 & 0.003 & 0.014 & Inconclusive \\
C6 & $\mathrm{mm}$ & 0.074 & 0.068 & 0.068 & App. Evolutionary \\
\hline FS5 & $\mathrm{mm}$ & 0.010 & 0.009 & 0.011 & App. Evolutionary \\
FS6 & $\mathrm{mm}$ & -0.036 & -0.036 & -0.040 & App. Evolutionary \\
\hline FS7 & $\mathrm{mm}$ & 0.083 & 0.082 & 0.050 & App. Evolutionary \\
\hline FS8 & $\mathrm{mm}$ & 0.002 & 0.002 & 0.002 & Stationary \\
\hline INC1 & $\circ$ & 0.007 & 0.007 & 0.007 & App. Evolutionary \\
\hline INC2 & $\circ$ & 0.028 & 0.026 & 0.031 & App. Evolutionary \\
\hline
\end{tabular}

The results indicate that the convergence extensometer placed across the southern transverse arch of 
the 4th bay is clearly experiencing an increasing trend of approximately $0.08 \mathrm{~mm} / \mathrm{year}$. The simpler methods considered also predict rates which are in good agreement with this. It can also be observed that the crackmeter placed across a crack in the vault of the central nave in the 7th bay is clearly stationary outside cyclic seasonal variations. Although there is a greater level of uncertainty associated to other estimated trends, the apparent evolutionary trend shown by the inclinometer monitoring the inclination of the front façade (INC2) deserves particular attention because despite being the sensor with the shortest duration of useful data (1.2 years), it shows a significant trend after the effect of temperature has been filtered out and the residuals from the models used for the filtering process have a relatively low scatter. In fact, it very nearly satisfies the condition to be classified as "Evolutionary" according to the procedure described in Section 3.2. Once again, evolution rates predicted by simpler methods are also in good agreement with the final one. The trend exhibited by this sensor corresponds to an outward inclination of the façade.

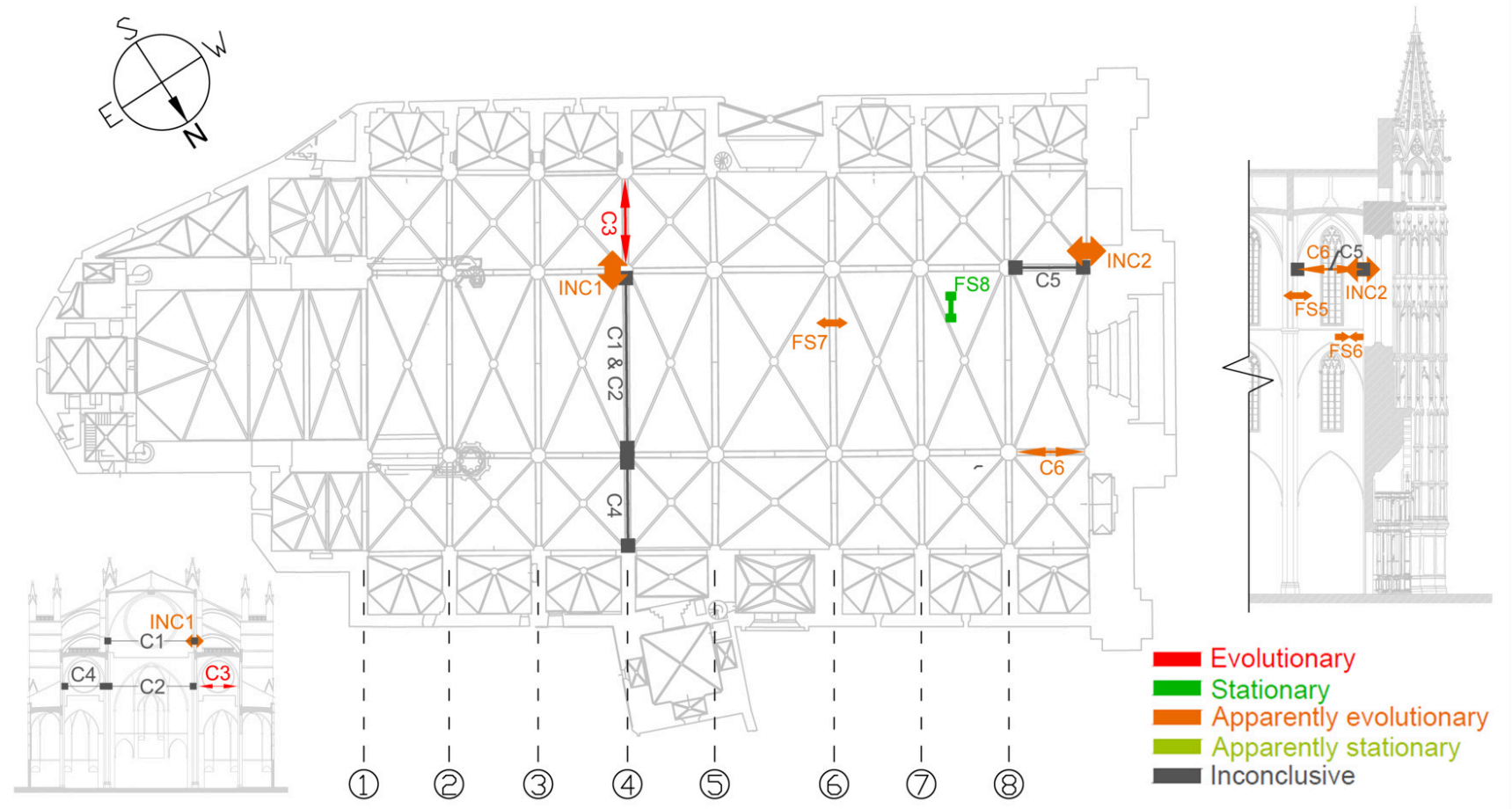

FIG URE 7 Main outcomes from analysis of static SHM data of Mallorca cathedral. (For interpretation of the references to colour in this figure, the reader is referred to the web version of this article)

\subsection{2 | Prognosis}

The opening trend across the southern transverse arches of Mallorca cathedral has been addressed in a previous study ${ }^{65}$ and can be attributed to a slow ongoing deformation caused by an unbalanced thrust during the construction process. The study, based on a time-dependent finite element analysis able to account for the creep behaviour of masonry, showed that the use of temporary ties during the construction process could clearly contribute to the structure's stability. The model used to represent the creep behaviour of masonry was calibrated based on a deformation rate of $0.1 \mathrm{~mm} /$ year between 543 and 548 years after construction (corresponding to the monitoring period) and concluded that under such conditions, this deformation would not have stabilised for a long period of time after construction. However, the research did indicate that if the model was calibrated for lower deformation rates, it could be shown that the phenomena would stabilise in a shorter time period.

The analysis of the monitoring data also reveals that the façade could be experiencing an outward inclination. The structure has already historically faced problems related to the main façade since the previous one was dismantled and reconstructed during the 19th century due to a worrying inclination. In fact, the out-of-plumb of the previous façade is recorded to have increased by $10 \mathrm{~cm}$ from the mid17 th century to the beginning of the 19th century. It should also be mentioned that a technical report based on an inspection carried out by the Spanish Institute of Cultural Heritage in $2012^{67}$ reports the presence of transverse cracks across the vault of the main entrance. Such damage would be consistent with problems related to the detachment of the façade. Moreover, an outward tilting of the façade would 
also be consistent with the apparent evolutionary trends derived for the convergence extensometer C6 and the crackmeters FS5 and FS7. However, it must also be said that it is unlikely that the entire façade is tilting outward as a rigid block because the magnitude of the estimated evolution rate is relatively high and hence such a phenomenon would have become clearly visible at a much earlier stage. It is more likely that only the upper part of the façade is experiencing an outward rotation with a rotating centre at a height between sensors FS5 and FS6. This would also explain the apparent underlying closing trend revealed for the crack monitored by FS6. These observations suggest that the façade is still being affected by an active mechanism and that further investigation could definitely shed more light on its true nature.

\section{2 | Monastery of Sant Cugat}

\subsection{1 | SHM system and results}

In order to investigate the root cause of several visible structural pathologies, a long-term static SHM system consisting of 14 crackmeters, 2 inclinometers, 3 thermistors and 3 humidity sensors was installed in the church of the monastery, as shown in Figure 9. Most of the sensors have been installed since March 2017 except the two crackmeters placed beneath the rose window (installed in December 2017) and the one placed in the lintel of the main entrance (installed in April 2018). In this case, the crackmeters and inclinometers have minimum resolutions of $0.003 \mathrm{~mm}$ and $0.002^{\circ}$ respectively. A summary of all the structural sensors of the system is given in Table 6. Although the system is still actively collecting data, the results presented in this paper were based on the data collected up to 22/06/2019. As such, Table 6 also shows the duration of useful data collected by each sensor up to this date.

TAB LE 6 Summary of structural sensors used in the SHM system installed in Sant Cugat monastery together with the total duration of useful data collected up to 22/06/2019 and estimated evolution rates from methods based on directly fitting time series to selected models.

\begin{tabular}{|c|cccc|c|c|}
\hline Sensor & Location & Years of data & Type & Units & \multicolumn{2}{c}{ Evolution rate [unit/year] } \\
\hline FS-1.1 & Fourth aisle & 2.3 & Crackmeter & $\mathrm{mm}$ & 0.085 & 0.078 \\
\hline FS-1.2 & & 2.3 & Crackmeter & $\mathrm{mm}$ & -0.002 & -0.001 \\
\hline INC-1.3 & Bell tower & 2.3 & Inclinometer & $\circ$ & 0.000 & -0.002 \\
\hline FS-2.5 & Sacristy & 2.3 & Crackmeter & $\mathrm{mm}$ & 0.020 & 0.017 \\
\hline FS-2.6 & & 2.3 & Crackmeter & $\mathrm{mm}$ & -0.006 & -0.004 \\
\hline FS-2.7 & Lateral aisle (bell tower) & 2.3 & Crackmeter & $\mathrm{mm}$ & -0.001 & 0.000 \\
\hline INC-2.8 & & 2.3 & Inclinometer & $\circ$ & -0.007 & -0.007 \\
\hline FS-2.11 & Central nave & 2.3 & Crackmeter & $\mathrm{mm}$ & 0.077 & 0.029 \\
\hline FS-3.15 & Central nave (exterior) & 2.3 & Crackmeter & $\mathrm{mm}$ & -0.028 & - \\
\hline FS-3.13 & & 2.3 & Crackmeter & $\mathrm{mm}$ & 0.012 & -0.023 \\
\hline FS-3.14 & Lateral aisle (monastery) & 2.3 & Crackmeter & $\mathrm{mm}$ & -0.026 & -0.052 \\
\hline FS-3.17 & & 2.3 & Crackmeter & $\mathrm{mm}$ & -0.044 & -0.055 \\
\hline FS-3.18 & Apse & 2.3 & Crackmeter & $\mathrm{mm}$ & -0.006 & 0.006 \\
\hline FS-3.19 & Interior front façade & 1.5 & Crackmeter & $\mathrm{mm}$ & 0.062 & 0.112 \\
\hline FS-3.20 & & 1.5 & Crackmeter & $\mathrm{mm}$ & -0.168 & -0.229 \\
\hline FS-3.21 & Lintel main entrance & 1.2 & Crackmeter & $\mathrm{mm}$ & 0.319 & 0.339 \\
\hline
\end{tabular}

In this case, the evolution rate estimated by the periodic nonlinear model for FS-3.15 was disregarded due to poor correlation with the measured data (coefficient of determination of 0.52). Once again, the predictions from both methods are in good agreement for certain sensors, notably for INC-2.8.

As for the previous case study, a comparison of the errors between measured responses and those simulated from linear and ARX models during their respective estimation phases was carried out in order to evaluate the ability of each model type to represent the dependency of structural parameters on environmental ones (see Figure 8). In this case, it is possible to see the added benefit of using both interior and exterior temperature as predictors in the ARX models since the MISO models outperform the SISO ones for almost all monitored responses despite the fact that they have lower model orders. 


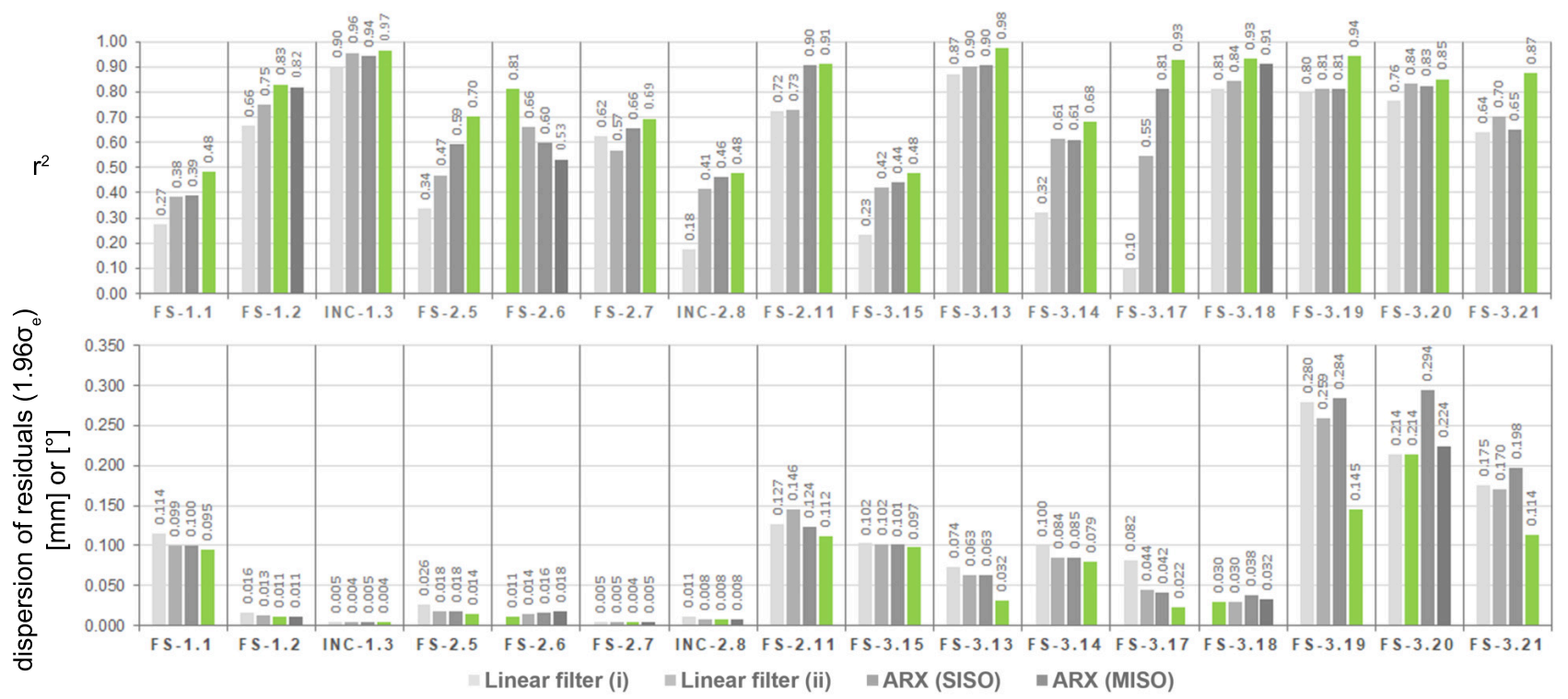

FIG URE 8 Coefficient of determination $\left(r^{2}\right)$ and dispersion of residuals $\left(1.96 \sigma_{e}\right)$ between simulated and measured responses in Sant Cugat monastery.

Before stating the main conclusions with respect to the diagnosis of the church's structural condition, the evolution rates estimated using methods described in Sections 2.3 and 3.1 as well as the evolutionary states evaluated from the procedure elaborated in Section 3.2 are summarised in Table 7 and Figure 9.

TAB LE 7 Comparison of estimated evolution rates for monitored structural parameters of Sant Cugat monastery from methods filtering out the simulated effect of measured environmental parameters.

\begin{tabular}{|cc|c|c|c|c|c|}
\hline \multirow{2}{*}{ Sensor } & \multirow{2}{*}{ Units } & \multicolumn{4}{|c|}{ Estimate of annual evolution rate [unit/year] } & \multirow{2}{*}{ Estimated condition } \\
\hline FS-1.1 & $\mathrm{mm}$ & 0.072 & 0.072 & 0.073 & 0.077 & App. Evolutionary \\
\hline FS-1.2 & $\mathrm{mm}$ & 0.003 & 0.003 & 0.003 & 0.003 & App. Evolutionary \\
\hline INC-1.3 & $\circ$ & -0.002 & -0.003 & -0.002 & -0.002 & Evolutionary \\
\hline FS-2.5 & $\mathrm{mm}$ & 0.016 & 0.016 & 0.015 & 0.017 & Evolutionary \\
\hline FS-2.6 & $\mathrm{mm}$ & -0.001 & -0.002 & -0.001 & 0.000 & App. Stationary \\
\hline FS-2.7 & $\mathrm{mm}$ & 0.000 & 0.000 & 0.000 & 0.000 & Stationary \\
\hline INC-2.8 & $\circ$ & -0.008 & -0.008 & -0.009 & -0.010 & Evolutionary \\
\hline FS-2.11 & $\mathrm{mm}$ & 0.045 & 0.042 & 0.054 & 0.040 & App. Evolutionary \\
\hline FS-3.15 & $\mathrm{mm}$ & -0.037 & -0.041 & -0.040 & -0.038 & App. Evolutionary \\
\hline FS-3.13 & $\mathrm{mm}$ & -0.023 & -0.022 & -0.025 & -0.025 & App. Evolutionary \\
\hline FS-3.14 & $\mathrm{mm}$ & -0.038 & -0.045 & -0.045 & -0.047 & App. Evolutionary \\
\hline FS-3.17 & $\mathrm{mm}$ & -0.048 & -0.052 & -0.047 & -0.063 & Inconclusive \\
\hline FS-3.18 & $\mathrm{mm}$ & 0.004 & 0.004 & -0.002 & 0.017 & App. Evolutionary \\
\hline FS-3.19 & $\mathrm{mm}$ & 0.033 & 0.036 & 0.038 & 0.099 & App. Evolutionary \\
\hline FS-3.20 & $\mathrm{mm}$ & -0.188 & -0.191 & -0.184 & -0.156 & App. Evolutionary \\
\hline FS-3.21 & $\mathrm{mm}$ & 0.157 & 0.148 & 0.151 & 0.087 & App. Evolutionary \\
\hline
\end{tabular}

The outcome of the analysis indicates that 3 of the monitored parameters are experiencing significant evolutionary trends outside the cyclic seasonal variations: the inclination of the bell tower (INC-1.3) as well as that of one of the pillars of the cimborio (INC-2.8) on the side of the bell tower towards the south and the opening of the crack in the Sacristy. All methods filtering out the simulated effect of environmental parameters predict very similar rates for these 3 trends. In fact for the inclination of the pillar of the cimborio, which shows the greatest predicted rate out of the two inclinations, methods based solely on fitting the time series to selected models also predict similar rates as those evaluated through the more sophisticated approaches. The same can be said about the crack in the Sacristy. As will be discussed in the next section, it is likely that these trends are being caused by the same phenomenon. 


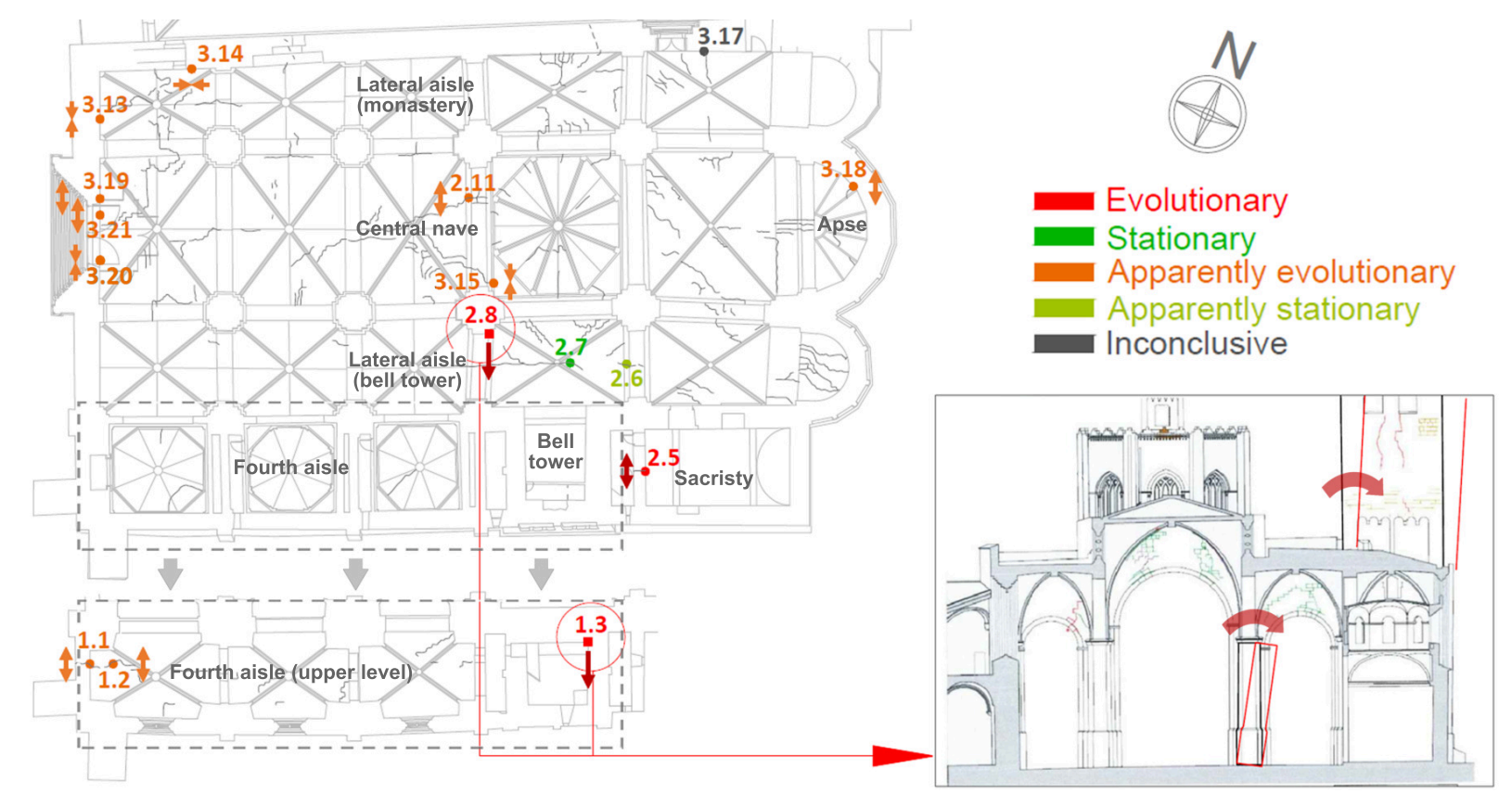

FIG URE 9 Main outcomes from analysis of static SHM data of Sant Cugat monastery. (For interpretation of the references to colour in this figure, the reader is referred to the web version of this article)

The results also reveal that the monitored crack across the vault of the lateral aisle appears to be stationary. It is interesting to note that in both case studies, the monitored parameter showing the most stationary trend corresponds to a crack in a vault. This is most probably due to the flexibility of vaults in comparison to other stiffer structural members and hence their increased ability to deform without suffering significant irreversible damage. It can also be seen that some of the monitored cracks in the lateral aisle on the side of the monastery appear to be experiencing closing trends outside seasonal variations. Since cracks are inherently caused due to the material experiencing tensile stresses, closing trends indicate that the behaviour of the structure has changed since the formation of the crack which initially had to be opening. It is interesting to note that the highest evolutionary rates are predicted for cracks in the interior of the front façade below the rose window. However, the magnitude of the observed trends are comparable to that of the errors associated to the models used to filter out the effect of temperature.

\subsection{2 | Prognosis}

The first conclusion that can be made from the analysis of the monitoring data so far is related to the effect of the bell tower on the rest of the elements. The measurements of the inclinometer on the wall of the bell tower suggest an outward leaning trend of $0.002^{\circ}$ year outside seasonal variations. The fact that the crack in the western wall of the sacristy appears to be opening at a rate of $0.017 \mathrm{~mm} /$ year is consistent with this movement since this wall is intrinsically tied to the bell tower. It also indicates that this outward rotation is most likely starting from a considerably low point (below the point at which the crack is already opening in the sacristy). Moreover, it is likely that the observed outward movement of the pillar supporting the cimborio is linked to this outward movement of the bell tower. All of these observations are consistent with the history of the construction of the structure since most of it was built in the 14th Century while the bell tower was only completed in the 18th Century, when an arch joining the then incomplete bell tower and the cimborio was also dismantled. It appears that the addition of this part of the structure is still having an active effect, even today. The bell tower has a total height of approximately $40 \mathrm{~m}$ and if this whole part was rotating outwards as a rigid block, the measured inclination would reflect an outward leaning of approximately $1.4 \mathrm{~cm}$ every 10 years at the top of the tower. In fact, recent topographic and laser scan surveys of the bell tower's geometry reveal that its southern and eastern wall have inclinations of up to $1.5 \%{ }^{68}$ corresponding to a net displacement of $52 \mathrm{~cm}$ from the vertical position at the top of the main body of the tower (see Figure 10). This strengthens the findings from the analysis of the monitoring data and suggests that a structural intervention could be required in the future to prevent further deterioration due to this mechanism. 

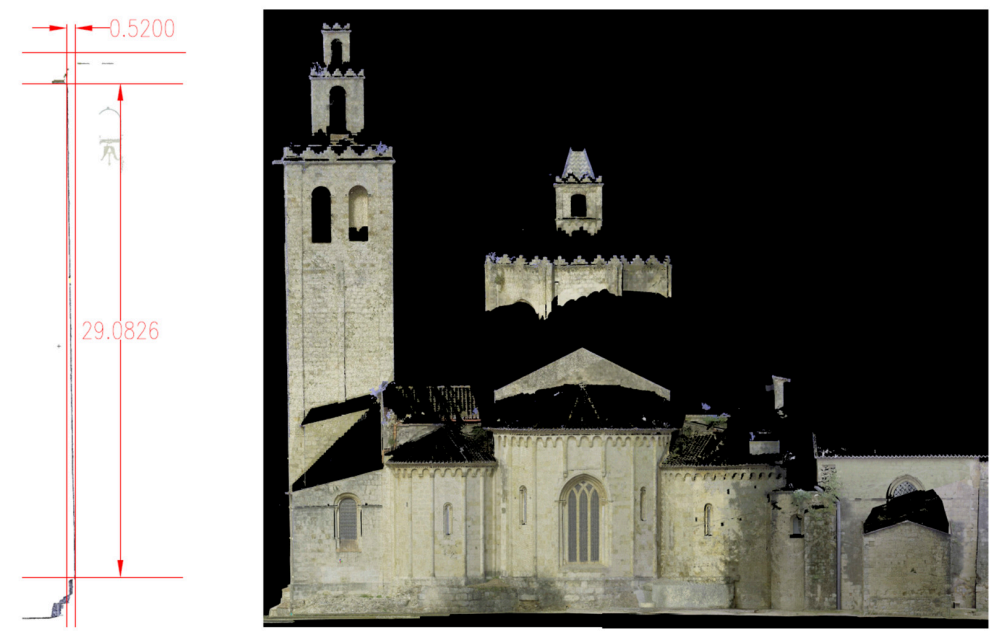

FIGURE 10 Measured inclination of the bell tower of Sant Cugat ${ }^{68}$.

With respect to the apparently evolutionary trends observed for cracks in the lateral aisle on the side of the monastery, these could possibly be caused by complex soil-structure interaction effects. In recent years, a new drainage system around the church has been installed and could help explain some of the closing trends observed for cracks next to the cloister. A longer monitoring period should help to shed more light on the true evolutionary nature and cause of these trends. Finally, the magnitude of the variations experienced by cracks below the rose window indicate that they deserve particular attention. However, since these cracks have been monitored for a shorter period of time and the fact that restoration works were being carried out on this part of the structure during the start of the monitoring period means that an acceptable level of confidence cannot yet be associated to any of the observed trends. Once again, an extended monitoring period is recommended before any suitable conclusions can be made on possible mechanisms affecting this area.

\section{5 | CONCLUSIONS}

This research has presented an integrated approach for the data analysis of static SHM of masonry heritage structures. The proposed methodology utilises dynamic linear regression models which can consider multiple predictors to filter out the reversible seasonal variations experienced by most structural parameters of interest. These models are able to attribute components of a current response caused by past ones when estimating the parameters of the model but can then disregard these components when simulating the effect only caused by environmental predictors. This is ideal for the case of static SHM since active structural mechanisms of interest have often begun long before any decision on monitoring could be made and it is thus impossible to define a period of time for which the relationship between structural and environmental parameters are isolated. In fact, the first part of the proposed method could also be used to filter out environmental effects on the evolution of natural frequencies recorded by dynamic monitoring systems. However, it is worth mentioning that in the case of masonry heritage structures, rather than attempting to detect very slow deterioration mechanisms, the dynamic monitoring strategy is most often oriented towards assessing the effectiveness of repairs, identifying significant changes in boundary conditions or towards the early identification of more pronounced damage characterised by a faster evolution rate. The nature of these objectives facilitates the application of several sophisticated analysis procedures because it simplifies the task of defining an adequate training period. As a result, several procedures including machine learning approaches, negative selection and principal component analysis could prove to be more efficient for the analysis of data from dynamic SHM systems.

The static SHM strategy is clearly well suited to identify slow-varying underlying trends in each monitored parameter. However, in most cases, cost and technical limitations only allow a very limited number of parameters to be monitored at specific location points. As a result, it can be very challenging to extract general conclusions on the global structural response. Given this inherent difficulty, one of the main advantages of the proposed methodology over previously applied ones is that it does not only provide estimated evolution rates of the monitored parameters but also evaluates their evolutionary state 
and classifies them accordingly. The classification is based on the estimated rates and the errors of the models used to represent the relationship between structural and environmental parameters. This can greatly help to identify areas that should be prioritised during the diagnosis of the structure and to extract meaningful conclusions on the relationship between different monitored parameters. In addition, the entire procedure can be fully automated and once implemented, can provide up to date analysis results as the monitoring period increases. In fact, an extension of the current research would be to assess if the trends of the residuals estimated after the proposed filtering of environmental effects change over longer monitoring periods. If they do, using higher order polynomial models to describe the trend could reveal whether or not a particular evolutionary state is stabilising or not.

The usefulness of the method has clearly been demonstrated through the application to two case studies. In both cases, outcomes of the proposed automatic procedure helped to identify vulnerable areas in important heritage structures. The results also reveal that simpler methods are often able to predict evolution rates rather accurately. This explains why such methods have been used successfully in the past for the accurate diagnosis of structures. However, such methods are not always accurate and provide very little means of assessing the reliability of results, whereas the proposed methodology is more robust and gives clear indications related to the reliability of results.

\section{ACKNOWLEDGEMENTS}

This research has received financial support from the City Council of Sant Cugat (Ajuntament de Sant Cugat) through a project aimed at monitoring the church of the Monastery of Sant Cugat (Estudi de monitorització i seguiment de l'estructura de l'Església del Monestir de Sant Cugat, ref. num. C-10764). Additional financial support has also been received from the Spanish Ministry of Education, Culture and Sports through a project aimed at studying the structural condition of Mallorca Cathedral (Estudio, diagnóstico y peritación y en su caso planteamiento de actuaciones sobre el comportamiento constructivo-estructural de la catedral de Santa María, en la ciudad de Palma, isla de Mallorca (Baleares), ref. num. 2/131400106ca - 5/030300592 EF). Support from the AGAUR agency of the Generalitat de Catalunya and the European Social Fund, in the form of a predoctoral grant awarded to the corresponding author is also gratefully acknowledged. Finally, the authors gratefully acknowledge the financial support from the Ministry of Science, Innovation and Universities of the Spanish Government, and the European Regional Development Fund through the SEVERUS project (Multilevel evaluation of seismic vulnerability and risk mitigation of masonry buildings in resilient historical urban centres, ref. num. RTI2018-099589-B-I00).

\section{CONFLICT OF INTEREST}

The authors declare that they have no conflict of interest.

\section{REFERENCES}

1. International Scientific Committee on the Analysis and Restoration of Structures of Architectural Heritage (ISCARSAH). Recommendations for the analysis, conservation and structural restoration of architectural heritage, 2005.

2. Ahmed Elyamani, Oriol Caselles, Pere Roca, and Jaime Clapes. Dynamic investigation of a large historical cathedral. Structural Control and Health Monitoring, 24(3):e1885, mar 2017.

3. Fuat Aras and Gulay Altay. Seismic evaluation and structural control of the historical Beylerbeyi Palace. Structural Control and Health Monitoring, 22(2):347-364, feb 2015.

4. Elisa Bassoli, Loris Vincenzi, Antonio Maria D'Altri, Stefano de Miranda, Marianna Forghieri, and Giovanni Castellazzi. Ambient vibration-based finite element model updating of an earthquake-damaged masonry tower. Structural Control and Health Monitoring, 25(5):e2150, may 2018.

5. Salvador Ivorra, Nicola Ivan Giannoccaro, and Dora Foti. Simple model for predicting the vibration transmission of a squat masonry tower by base forced vibrations. Structural Control and Health Monitoring, 26(6):e2360, jun 2019.

6. A. G. El-Attar, A. M. Saleh, and A. H. Zaghw. Conservation of a slender historical Mamluk-style minaret by passive control techniques. Structural Control and Health Monitoring, 12(2):157-177, apr 2005. 
7. Salvatore Russo and Eleonora Spoldi. Damage assessment of Nepal heritage through ambient vibration analysis and visual inspection. Structural Control and Health Monitoring, feb 2020.

8. Filippo Lorenzoni. Integrated methodologies based on Structural Health Monitoring for the protection of Cutural Heritage buildings. Phd thesis, University of Trento, 2013.

9. H. Shi, K. Worden, and E. J. Cross. A nonlinear cointegration approach with applications to structural health monitoring. Journal of Physics: Conference Series, 744(1), 2016.

10. Alberto Barontini, Maria Giovanna Masciotta, Luís F. Ramos, Paulo Amado Mendes, and Paulo B. Lourenço. Application of a bio-inspired anomaly detection algorithm for unsupervised SHM of a historic masonry church. In 10th International Masonry Conference (10th IMC), Milan, Italy, 2018.

11. Nguyen Lu Dang Khoa, Mehrisadat Makki Alamdari, Thierry Rakotoarivelo, Ali Anaissi, and Yang Wang. Structural Health Monitoring Using Machine Learning Techniques and Domain Knowledge Based Features. In Human and Machine Learning, pages 409-435. Springer, Cham, 2018.

12. Riccardo Mario Azzara, Guido De Roeck, Maria Girardi, Cristina Padovani, Daniele Pellegrini, and Edwin Reynders. The influence of environmental parameters on the dynamic behaviour of the San Frediano bell tower in Lucca. Engineering Structures, 156(March 2017):175-187, feb 2018.

13. Alessandro Cabboi, Carmelo Gentile, and Antonella Saisi. From continuous vibration monitoring to FEM-based damage assessment: Application on a stone-masonry tower. Construction and Building Materials, 156:252-265, dec 2017.

14. A. Deraemaeker and K. Worden. A comparison of linear approaches to filter out environmental effects in structural health monitoring. Mechanical Systems and Signal Processing, 105:1-15, may 2018.

15. F.J. Baeza, Salvador Ivorra, D. Bru, and F.B. Varona. Structural Health Monitoring Systems for Smart Heritage and Infrastructures in Spain. In Erika Ottaviano, Assunta Pelliccio, and Vincenzo Gattulli, editors, Mechatronics for Cultural Heritage and Civil Engineering, volume 92 of Intelligent Systems, Control and Automation: Science and Engineering, pages 271-294. Springer International Publishing, 2018.

16. Bram Cornelis and Bart Peeters. Online Bayesian spike removal algorithms for structural health monitoring of vehicle components. In Eurodyn 2014: Proceedings of the 9th International Conference on Structural Dynamics, pages 2295-2301, Porto, jul 2014.

17. Andrew Halfpenny. Data Preprocessing for Damage Detection. In Encyclopedia of Structural Health Monitoring. John Wiley \& Sons, Ltd, Chichester, UK, jan 2008.

18. Simonetta Baraccani, Michele Palermo, Riccardo M. Azzara, Giada Gasparini, Stefano Silvestri, and Tomaso Trombetti. Structural Interpretation of Data from Static and Dynamic Structural Health Monitoring of Monumental Buildings. Key Engineering Materials, 747:431-439, jul 2017.

19. Keith Worden, Charles R. Farrar, Graeme Manson, and Gyuhae Park. The fundamental axioms of structural health monitoring. Proceedings of the Royal Society A: Mathematical, Physical and Engineering Sciences, 463(2082):1639-1664, 2007.

20. Hoon Sohn. Effects of environmental and operational variability on structural health monitoring. Philosophical Transactions of the Royal Society A: Mathematical, Physical and Engineering Sciences, 365(1851):539-560, feb 2007.

21. Gianni Bartoli, Andrea Chiarugi, and Vittorio Gusella. Monitoring Systems on Historic Buildings: The Brunelleschi Dome. Journal of Structural Engineering, 122(6):663-673, jun 1996.

22. Federica Ottoni and Carlo Blasi. Results of a 60-Year Monitoring System for Santa Maria del Fiore Dome in Florence. International Journal of Architectural Heritage, 9(1):7-24, jan 2015.

23. Pier Paolo Rossi and Christian Rossi. Monitoring of Two Great Venetian Cathedrals: San Marco and Santa Maria Gloriosa Dei Frari. International Journal of Architectural Heritage, 9(1):58-81, jan 2015.

24. Abraham R. Sánchez, Roberto Meli, and Marcos M. Chávez. Structural Monitoring of the Mexico City Cathedral (1990-2014). International Journal of Architectural Heritage, 10(2-3):15583058.2015.1113332, dec 2015.

25. Simonetta Baraccani, Stefano Silvestri, Giada Gasparini, Michele Palermo, Tomaso Trombetti, Elena Silvestri, Renato Lancellotta, and Alessandro Capra. A Structural Analysis of the Modena Cathedral. International Journal of Architectural Heritage, 10(23):15583058.2015.1113344, dec 2015. 
26. Rosario Ceravolo, Annunziata De Marinis, Marica L. Pecorelli, and Luca Zanotti Fragonara. Monitoring of masonry historical constructions: 10 years of static monitoring of the world's largest oval dome. Structural Control and Health Monitoring, 24(10):e1988, oct 2017.

27. Maria-Giovanna Masciotta, João C.A. Roque, Luís F. Ramos, and Paulo B. Lourenço. A multidisciplinary approach to assess the health state of heritage structures: The case study of the Church of Monastery of Jerónimos in Lisbon. Construction and Building Materials, 116:169-187, jul 2016.

28. Filippo Lorenzoni, Filippo Casarin, Claudio Modena, Mauro Caldon, Kleidi Islami, and Francesca da Porto. Structural health monitoring of the Roman Arena of Verona, Italy. Journal of Civil Structural Health Monitoring, 3(4):227-246, dec 2013.

29. Haydee Blanco, Yosbel Boffill, Ignacio Lombillo, and Luis Villegas. An integrated structural health monitoring system for determining local/global responses of historic masonry buildings. Structural Control and Health Monitoring, 25(8):e2196, aug 2018.

30. F. Lucchin G. Gaudini, C. Modena, F. Casarin, C. Bettio. Monitoring and strengthening interventions on the stone tomb of Cansignorio della Scala, Verona, Italy. In Proceedings of the VI International Conference on Structural Analysis of Historic Construction, SAHC08, pages 423-432. CRC Press, jun 2008.

31. Salvatore Russo. On the monitoring of historic Anime Sante church damaged by earthquake in L'Aquila. Structural Control and Health Monitoring, 20(9):1226-1239, sep 2013.

32. A. J. Lawrance. Directionality and Reversibility in Time Series. International Statistical Review / Revue Internationale de Statistique, 59(1):67, apr 1991.

33. Simonetta Baraccani, Tomaso Trombetti, Michele Palermo, Giada Gasparini, Stefano Silvestri, and Antoine Dib. A Methodology of Analysis for a Critique Interpretation of the Data Acquired from Monitoring Systems of Historical Buildings. In 7th European Workshop on Structural Health Monitoring, 2014.

34. Mahayaudin M. Mansor, Max E. Glonek, David A. Green, and Andrew V. Metcalfe. Threshold Autoregressive Models for Directional Time Series. In Time Series Analysis and Forecasting. Contributions to Statistics., pages 13-25. Springer, Cham, 2016.

35. Elizabeth J. Cross, Keith Worden, and Qian Chen. Cointegration: a novel approach for the removal of environmental trends in structural health monitoring data. Proceedings of the Royal Society A: Mathematical, Physical and Engineering Sciences, 467(2133):2712-2732, sep 2011.

36. Edwin Reynders, Gersom Wursten, and Guido de Roeck. Output-only structural health monitoring in changing environmental conditions by means of nonlinear system identification. Structural Health Monitoring, 13(1):82-93, 2014.

37. Bart Peeters and Guido De Roeck. One-year monitoring of the Z24-Bridge: environmental effects versus damage events. Earthquake Engineering \& Structural Dynamics, 30(2):149-171, feb 2001.

38. L.F. Ramos, L. Marques, P.B. Lourenço, G. De Roeck, A. Campos-Costa, and J. Roque. Monitoring historical masonry structures with operational modal analysis: Two case studies. Mechanical Systems and Signal Processing, 24(5):1291-1305, jul 2010.

39. Alban Kita, Nicola Cavalagli, and Filippo Ubertini. Temperature effects on static and dynamic behavior of Consoli Palace in Gubbio, Italy. Mechanical Systems and Signal Processing, 120:180-202, apr 2019.

40. Filippo Lorenzoni, Filippo Casarin, Mauro Caldon, Kleidi Islami, and Claudio Modena. Uncertainty quantification in structural health monitoring: Applications on cultural heritage buildings. Mechanical Systems and Signal Processing, 66-67:268-281, jan 2016.

41. Bart Peeters. System Identification and Damage Detection in Civil Engineering. Phd thesis, KU Leuven, 2000.

42. Maria-Giovanna Masciotta, Luis F. Ramos, Paulo B. Lourenço, and José A. C. Matos. Development of Key Performance Indicators for the Structural Assessment of Heritage Buildings. In 8th European Workshop on Structural Health Monitoring (EWSHM 2016), 5-8 July 2016, Bilbao, Spain, pages 5-8, jul 2016.

43. Jorge J. Moré. The Levenberg-Marquardt algorithm: Implementation and theory. In G. A. Watson, editor, Numerical Analysis, pages 105-116. Springer, Berlin, Heidelberg, 1978.

44. J Meeus and D Savoie. The history of the tropical year. Journal of the British Astronomical Association, 102:40-42, 1992.

45. Ingrid Daubechies. Ten Lectures on Wavelets. Society for Industrial and Applied Mathematics, jan 1992. 
46. David L. Donoho and lain M. Johnstone. Ideal Spatial Adaptation by Wavelet Shrinkage. Biometrika, 81(3):425, aug 1994.

47. A. G. Asuero, A. Sayago, and A. G. González. The Correlation Coefficient: An Overview. Critical Reviews in Analytical Chemistry, 36(1):41-59, jan 2006.

48. Åke Björck. Solving linear least squares problems by Gram-Schmidt orthogonalization. BIT, 7(1):1-21, mar 1967.

49. Amde M Amde, J V Martin, and James Colville. The Effects of Moisture on Compressive Strength and Modulus of Brick Masonry. In 13th International Brick and Block Masonry Conference, Amsterdam, 2007.

50. Navaratnarajah Sathiparan and Udayakumar Rumeshkumar. Effect of moisture condition on mechanical behavior of low strength brick masonry. Journal of Building Engineering, 17(February):23-31, may 2018.

51. Jiri Witzany, Tomas Cejka, and Radek Zigler. The Effect of Moisture on Significant Mechanical Characteristics of Masonry. Engineering Structures and Technologies, 2(3):79-85, sep 2010.

52. Antonia Moropoulou, Maria Karoglou, loannis Agapakis, Charalampos Mouzakis, Simos Asimakopoulos, George Pantazis, and Evangelia Lambrou. Structural health monitoring of the Holy Aedicule in Jerusalem. Structural Control and Health Monitoring, 26(9), sep 2019.

53. Luís F Ramos. Damage identification on masonry structures based on vibration signatures. Phd thesis, Universidade do Minho, 2007.

54. Daniele Ferretti and Zdeněk P. Bažant. Stability of ancient masonry towers: Moisture diffusion, carbonation and size effect. Cement and Concrete Research, 36(7):1379-1388, jul 2006.

55. D. G. Toll, Z. Abedin, J. Buma, Y. Cui, A. S. Osman, and K.K. Phoon. The impact of changes in the water table and soil moisture on structural stability of buildings and foundation systems : systematic review CEE10-005 (SR90). Technical report, Collaboration for Environmental Evidence, 2012.

56. M.J. DeJong. Settlement effects on masonry structures. In Structural Analysis of Historical Constructions: Anamnesis, diagnosis, therapy, controls - Proceedings of the 10th International Conference on Structural Analysis of Historical Constructions, SAHC 2016, pages 449-456, 2016.

57. Carl. De Boor. A Practical Guide to Splines. Springer-Verlag New York, 1978.

58. F. N. Fritsch and R. E. Carlson. Monotone Piecewise Cubic Interpolation. SIAM Journal on Numerical Analysis, 17(2):238-246, apr 1980.

59. Hugues Garnier and Liuping Wang. Identification of Continuous-time Models from Sampled Data. Advances in Industrial Control. Springer London, London, 2008.

60. Roberto Diversi, Roberto Guidorzi, and Umberto Soverini. Identification of ARX and ARARX Models in the Presence of Input and Output Noises. European Journal of Control, 16(3):242-255, jan 2010.

61. Stijn Verbeke and Amaryllis Audenaert. Thermal inertia in buildings: A review of impacts across climate and building use. Renewable and Sustainable Energy Reviews, 82(September 2017):2300-2318, feb 2018.

62. Koray Ulgen. Experimental and theoretical investigation of effects of wall's thermophysical properties on time lag and decrement factor. Energy and Buildings, 34(3):273-278, mar 2002.

63. Gary Smith. Simple Regression. In Essential Statistics, Regression, and Econometrics, pages 219-258. Elsevier, 2012.

64. Hubert W. Lilliefors. On the Kolmogorov-Smirnov Test for Normality with Mean and Variance Unknown. Journal of the American Statistical Association, 62(318):399, jun 1967.

65. Luca Pelà, Julien Bourgeois, Pere Roca, Miguel Cervera, and Michele Chiumenti. Analysis of the Effect of Provisional Ties on the Construction and Current Deformation of Mallorca Cathedral. International Journal of Architectural Heritage, 10(4):418-437, may 2016.

66. Pere Roca, Miguel Cervera, Luca Pelà, Roberto Clemente, and Michele Chiumenti. Continuum FE models for the analysis of Mallorca Cathedral. Engineering Structures, 46:653-670, jan 2013.

67. IPCE: Instituto del Patrimonio Cultural de Esapaña (Spanish Institute of Cultural Heritage). Inspección técnica portadas de la Catedral de Palma de Mallorca (Technical inspection of the portal of Palma de Mallorca cathedral). Technical report, aug 2012.

68. Ajuntament de Sant Cugat (Sant Cugat City Council). Informe tècnic: Comprovació de la verticalitat de la torre del campanar del monestir (Measurement of the inclination of the bell tower of the monastery). Technical report, 2019. 